\title{
The mitochondrial $\mathrm{Na}^{+} / \mathrm{Ca}^{2+}$ exchanger is essential for $\mathrm{Ca}^{2+}$ homeostasis and viability
}

\author{
Timothy S. Luongo ${ }^{1}$, Jonathan P. Lambert ${ }^{1}$, Polina Gross ${ }^{2}$, Mary Nwokedi ${ }^{1}$, Alyssa A. \\ Lombardi $^{1}$, Santhanam Shanmughapriya ${ }^{1}$, April C. Carpenter ${ }^{3}$, Devin Kolmetzky ${ }^{1}$, Erhe \\ Gao ${ }^{1}$, Jop H. van Berlo ${ }^{4}$, Emily J. Tsai ${ }^{5}$, Jeffery D. Molkentin ${ }^{6}$, Xiongwen Chen ${ }^{2}$, \\ Muniswamy Madesh ${ }^{1}$, Steven R. Houser ${ }^{2}$, and John W. Elrod ${ }^{1}$ \\ ${ }^{1}$ Center for Translational Medicine, Department of Pharmacology, Temple University School of \\ Medicine, Philadelphia, Pennsylvania 19140, USA \\ ${ }^{2}$ Cardiovascular Research Center, Department of Physiology, Temple University School of \\ Medicine, Philadelphia, Pennsylvania 19140, USA \\ ${ }^{3}$ Department of Health and Exercise Physiology, Ursinus College, Collegeville, Pennsylvania \\ 19426, USA \\ ${ }^{4}$ Department of Medicine, University of Minnesota, Minneapolis, Minnesota 55455, USA \\ ${ }^{5}$ Division of Cardiology, Department of Medicine, College of Physicians \& Surgeons, Columbia \\ University, New York, New York 10032, USA \\ ${ }^{6}$ Department of Pediatrics, University of Cincinnati, Cincinnati Children's Hospital Medical Center, \\ Howard Hughes Medical Institute, Cincinnati, Ohio 45229, USA
}

\section{Abstract \\ Mitochondrial calcium $\left(\mathrm{m}^{2+}\right)$ has a central role in both metabolic regulation and cell death signalling, however its role in homeostatic function and disease is controversial ${ }^{1}$. Slc8b1 encodes the mitochondrial $\mathrm{Na}^{+} / \mathrm{Ca}^{2+}$ exchanger (NCLX), which is proposed to be the primary mechanism for $\mathrm{mCa}^{2+}$ extrusion in excitable cells ${ }^{2,3}$. Here we show that tamoxifen-induced deletion of $S / c 8 b 1$}

Reprints and permissions information is available at www.nature.com/reprints.

Correspondence and requests for materials should be addressed to J.W.E. (elrod@temple.edu).

Supplementary Information is available in the online version of the paper.

Author Contributions J.W.E. and T.S.L. contributed to study design, data analysis and writing the paper; T.S.L. was involved with all assays and data collection; J.P.L. performed western blots and NADH assays; P.G. performed ACM $\mathrm{iCa}^{2+}$ transient analysis; M.N. performed myocardial infarction data acquisition; A.A.L. assisted with the ischaemia reperfusion study and mitochondrial membrane potential assays; S.S. and M.M. assisted with efflux analysis in permeabilized cells; A.C.C. performed qPCR assays; D.K. performed histology; E.G. performed ischaemia reperfusion and myocardial infarction surgeries; J.H.v.B. and J.D.M. aided mutant mouse generation; E.J.T. supplied human heart samples; X.C. performed radiotelemeter implantation and assisted with electrocardiogram analysis; J.W.E., J.H.v.B, T.S.L., S.R.H., J.P.L., A.C.C. and A.A.L. assisted with data interpretation and edited the manuscript.

The authors declare no competing financial interests.

Readers are welcome to comment on the online version of the paper.

Publisher's note: Springer Nature remains neutral with regard to jurisdictional claims in published maps and institutional affiliations.

Reviewer Information Nature thanks M. Murphy and the other anonymous reviewer(s) for their contribution to the peer review of this work.

Online Content Methods, along with any additional Extended Data display items and Source Data, are available in the online version of the paper; references unique to these sections appear only in the online paper. 
in adult mouse hearts causes sudden death, with less than $13 \%$ of affected mice surviving after 14 days. Lethality correlated with severe myocardial dysfunction and fulminant heart failure. Mechanistically, cardiac pathology was attributed to $\mathrm{m}^{2} \mathrm{C}^{2+}$ overload driving increased generation of superoxide and necrotic cell death, which was rescued by genetic inhibition of mitochondrial permeability transition pore activation. Corroborating these findings, overexpression of NCLX in the mouse heart by conditional transgenesis had the beneficial effect of augmenting $\mathrm{ma}^{2+}$ clearance, preventing permeability transition and protecting against ischaemia-induced cardiomyocyte necrosis and heart failure. These results demonstrate the essential nature of $\mathrm{mCa}^{2+}$ efflux in cellular function and suggest that augmenting $\mathrm{maa}^{2+}$ efflux may be a viable therapeutic strategy in disease.

The genes regulating mitochondrial calcium exchange have only recently been identified and most have not been functionally evaluated in vivo ${ }^{4-10}$. Perhaps the most suited and studied system of $\mathrm{mCa}^{2+}$ exchange is the cardiomyocyte, which contains a significant number of mitochondria (around 35\% of total cell volume) and wherein intracellular $\mathrm{Ca}^{2+}\left(\mathrm{Ca}^{2+}\right)$ levels are high and vary greatly on a beat-to-beat basis ${ }^{11,12}$. It was recently reported by our group and others that inhibition of acute ${ }_{\mathrm{m}} \mathrm{Ca}^{2+}$ uptake by deletion of the $\mathrm{Mcu}$ gene caused a loss of stress responsive signalling, but surprisingly did not give rise to a basal phenotype ${ }^{13-15}$. These observations, coupled with the findings that resting $\mathrm{mCa}^{2+}$ levels were unchanged in $\mathrm{Mcu}$ knockout models suggest that an as yet undiscovered MCUindependent uptake mechanism probably compensates to support normal mitochondrial function, but is insufficient for the range of $\mathrm{m}^{2+}$ signalling required for cardiac adaptation during stress ${ }^{13}$. Given that ${ }_{\mathrm{m}} \mathrm{Ca}^{2+}$ uptake must be balanced with extrusion in the homeostatic condition, we hypothesized that targeting the predominant ${ }_{\mathrm{m}} \mathrm{Ca}^{2+}$ efflux mechanism, NCLX, would be sufficient to perturb the $\mathrm{ma}^{2+}$ pool and aid our understanding of $\mathrm{m}^{\mathrm{Ca}^{2+}}$ signalling and its role in health and disease $\mathrm{e}^{2,16,17}$.

We verified that genetic inhibition of NCLX in a cell line abrogated $\mathrm{mCa}^{2+}$ efflux (Extended Data Fig. 1) and we generated a conditional loss-of-function mouse model wherein exons 57 of $S / c 8 b 1$ are flanked by $l o x P \operatorname{sites}^{18}$ (Slc $8 b 1^{f l / f l}$, Fig. 1a; Methods). $S I c 8 b 1^{f l / f l}$ mice were cross-bred with the tamoxifen-inducible, aMHC-MerCreMer (MCM) transgenic model to allow cardiomyocyte-specific deletion in the adult heart ${ }^{19}$. We confirmed an approximate $70 \%$ loss of NCLX protein three days after tamoxifen administration (Fig. 1b). This level of protein loss is as expected, owing to the mosaic Cre expression in this model ${ }^{20}$. There was little difference in the expression of other proposed $\mathrm{mCa}^{2+}$ regulators, although we noted a decrease in the MCU-associated protein, $\mathrm{EMRE}^{10}$, suggesting a possible compensatory alteration to inhibit ${ }_{\mathrm{m}} \mathrm{Ca}^{2+}$ uptake (Fig. 1 b). $87 \%$ of $S 1 \mathrm{c} 8 \mathrm{~b} 1$ conditional knockout mice died within two weeks of tamoxifen delivery (Fig. 1c). This notable result suggests that NCLXmediated efflux is necessary to maintain homeostatic $\mathrm{mCa}^{2+}$ levels in cardiomyocytes and is necessary for survival. Echocardiographic analysis three days post tamoxifen revealed left ventricular dilation and decreased left ventricular function (Fig. 1d, e; Extended Data Fig. 2c). Gross examination of NCLX-knockout hearts revealed substantial cardiac remodelling (Fig. 1f, g), including an increase in heart mass and correlative change in cardiomyocyte cross-sectional area (Fig. 1h, i), and a significant increase in cardiac fibrosis (Fig. 1j, k). 
To assess the mechanism whereby acute deletion of $S / c 8 b 1$ in adult cardiomyocytes (ACMs) elicited heart failure and sudden death, we isolated cardiac mitochondria and evaluated mitochondrial swelling, an indicator of mitochondrial permeability transition pore (MPTP) opening. Mitochondria isolated from $S l c 8 b 1$ conditional knockout hearts swelled to a greater extent and at a faster rate than controls (Fig. 2a, b; Extended Data Fig. 2d). Since mitochondria isolated from $S l c 8 b 1^{f l / f l} \times$ MCM hearts were larger at baseline, we added polyethylene glycol (PEG) to osmotically shrink mitochondria and control for possible alterations in mitochondrial morphology. Following PEG addition, mitochondria isolated from $S l c 8 b 1^{f l / f l} \times \mathrm{MCM}$ hearts returned to the same size as controls suggesting they were pre-swollen, indicative of baseline MPTP activity and $\mathrm{Ca}^{2+}$ overload (Fig. 2c, d). Given that $\mathrm{m}^{2+}$ overload is a mechanism for substantial increases in electron-transport-chaingenerated superoxide ${ }^{21}$, we examined reactive oxygen species (ROS) production in live left ventricular tissue sections and quantified a 4.2-fold increase in superoxide generation in Slc8b1-null cardiomyocytes versus controls (Fig. 2e, f). To examine redox stress further, we isolated ACMs and monitored mitochondrial superoxide production (Extended Data Fig. 2e, f). While isolating intact ACMs with normal sarcomeric structure three days after tamoxifen-induced Slc8b1 deletion proved challenging, we did find a significant increase in mitochondrial superoxide generation. MPTP activation and elevated ROS are key mechanisms that initiate cellular necrosis. To survey active necrosis, we employed an Evans blue dye (EBD) exclusion technique whereby circulating EBD bound to albumin can only enter cardiomyocytes with ruptured sarcolemma (Fig. 2g). We observed compromised sarcolemmal integrity in $8 \%$ of cardiomyocytes in Slc8b1 conditional knockout hearts, two days after tamoxifen administration (Fig. 2h). These data confirm substantial cardiomyocyte death immediately after ablating $\mathrm{m}^{2+}$ efflux, suggesting that ${ }_{\mathrm{m}} \mathrm{Ca}^{2+}$ overload is the pathogenic stimulus. To further define the mechanism underlying the sudden death phenotype following the acute deletion of $S \mid c 8 b 1$, we continuously recorded electrocardiograms in conscious $S I c 8 b 1$ conditional knockout mice before and following tamoxifen administration. Arrhythmic events were only recorded immediately before sinus arrest and were characterized by prolongation of the PR interval, QRS interval widening, and bradycardia (Extended Data Fig. 2g-j and Extended Data Table 1). These results indicate that the cumulative loss of cardiomyocytes and replacement fibrosis precedes the organ-level conduction derangement. To definitively examine if the cardiac failure and sudden lethality induced by NCLX deficiency was due to mitochondrial-dependent necrosis, we crossed NCLX conditional knockout mice with the cyclophilin D (CypD)-null mouse $\left(\right.$ Ppif $\left.^{--}\right)$. CypD is the only genetically confirmed component of the MPTP, and CypD inhibition impedes MPTP activation in response to $\mathrm{Ca}^{2+}$ and ROS stress ${ }^{22}$. Depletion of CypD on the NCLX conditional knockout background rescued lethality following tamoxifen administration, with $63 \%$ of double-knockout mice $\left(\right.$ Slc8b1 $1^{f l / f l} \times \mathrm{MCM} \times$ Ppif $^{\prime-}$ ) surviving, compared to only a single NCLX conditional knockout (Fig. 2i). Loss of CypD elicited near complete rescue of left ventricular function (Fig. 2j) and hypertrophy (Fig. 2k).

Transmission electron microscopy visualization of NCLX-null cardiomyocyte ultrastructure confirmed substantial sarcomere disorganization, which was rescued by depletion of CypD (Fig. 21). Examination of mitochondrial cristae density, a hallmark of MPTP activation and swelling, revealed an approximate fourfold decrease in NCLX conditional knockout hearts, which was restored to near control levels in CypD double-knockout mice (Fig. 2m). We also 
quantified an increase in the number of both fragmented and enlarged mitochondria in Slc $8 b 1^{f l / f l} \times \mathrm{MCM}$ hearts, which was also corrected with deletion of CypD (Extended Data Fig. 2i).

The rampant $\mathrm{mCa}^{2+}$ overload and substantial necrotic cell death caused by acute deletion of NCLX from the adult heart made it impossible to examine $\mathrm{m}^{2+}$ dynamics in this model. Further, $\mathrm{Ca}^{2+}$ dynamics change rapidly in response to stress; therefore, to maintain focus on the physiological function of NCLX, we crossed $S l c 8 b 1^{f l / f l}$ mice with a constitutively active aMHC-Cre model, wherein Cre-mediated recombination occurs shortly after birth ${ }^{23}$. Early deletion allowed for undefined compensatory adaptations in the maturing heart resulting in normal viability (Extended Data Table 2). Mice were injected with AAV6 encoding a mitochondrial-targeted $\mathrm{Ca}^{2+}$ reporter before ACM isolation and imaged at a slow rate of pacing $(0.1 \mathrm{~Hz})$ to resolve individual $\mathrm{m}^{2+}$ transients (Fig. 2n; Extended Data Fig. 3b, c). Deletion of Slc8b1 did not alter the peak amplitude (Fig. 2o), rate of uptake (Extended Data Fig. 3d), or mitochondrial membrane potential ( $\Delta \psi$, Extended Data Fig. 3f), but did elicit a near-complete loss of $\mathrm{m}^{\mathrm{Ca}^{2+}}$ efflux capacity (Fig. 2p; Extended Data Fig. 3e). In addition, Slc8b1 deletion in cardiomyocytes had no significant impact on cytosolic ${ }_{\mathrm{i}} \mathrm{Ca}^{2+}$ cycling (Extended Data Fig. 3g-1). This is not surprising, as cardiac mitochondria are not believed to serve as a significant ${ }_{i} \mathrm{Ca}^{2+}$ buffer under physiological conditions ${ }^{24}$. Monitoring $\mathrm{m}_{\mathrm{Ca}}{ }^{2+}$ efflux independent of MCU-mediated uptake, with inhibition of sarcoplasmic reticulum and sarcolemmal $\mathrm{Ca}^{2+}$ transport, revealed a $67 \%$ reduction in the rate of $\mathrm{mCa}^{2+}$ efflux in $S l c 8 b 1^{-/-}$ACMs (Fig. 2q-u). This reduction in extrusion capacity corresponded to the degree of inhibition elicited by pharmacological NCLX inhibition in wild-type cardiomyocytes (Fig. 2t). In addition to the loss of $\mathrm{m}^{2} \mathrm{a}^{2+}$ efflux, we found a significant reduction in $\mathrm{mCa}^{2+}$ uptake (Extended Data Fig. 3m, n). Since we did not observe major changes in the expression of MCU components (Extended Data Fig. 3a), we attribute this decrease in uptake to compensatory alterations in MCU regulation. We suspect that this adaptation may contribute to the difference in survival seen with early NCLX deletion versus acute deletion in the adult heart (Extended Data Table 2). While no change in ${ }_{\mathrm{m}} \mathrm{Ca}^{2+}$ uptake was seen in paced ACMs (Fig. 2o; Extended Data Fig. 3d), we hypothesize that it manifested in this reductionist system owing to the higher level of bath $\mathrm{Ca}^{2+}(20 \mu \mathrm{M})$. We suspect that NCLX deletion in the maturing myocardium (aMHC-Cre model) allows for a counterbalancing decrease in $\mathrm{mCa}^{2+}$ uptake. We have discounted that loss of NCLX limits $\mathrm{mCa}^{2+}$ uptake (reverse-mode exchange), as experimentally we find that acute knockdown, mediated by short hairpin RNA, or CGP inhibition of NCLX enhances $\mathrm{mCa}^{2+}$ accumulation (Fig. 2t; Extended Data Fig. 1a-c).

Dysregulation of ${ }_{\mathrm{i}} \mathrm{Ca}^{2+}$ cycling is a prominent feature of heart failure development and progression ${ }^{25}$. However, whether $\mathrm{mCa}^{2+}$ exchange contributes to disease progression remains controversial ${ }^{26-28}$. Genetic deletion of $\mathrm{Mcu}$, the pore forming component of the uniporter, had no effect on pressure-overload-induced heart failure ${ }^{15,29}$, calling into question the relevance of $\mathrm{mCa}^{2+}$ exchange during chronic stress. To investigate this further, we measured the expression of $\mathrm{mCa}^{2+}$-associated genes in left ventricular biopsies of explanted failing hearts of transplant recipients. We discovered that the expression of SLCSB1 and MICU1, a negative regulator of MCU activity at $\operatorname{low}_{\mathrm{i}} \mathrm{Ca}^{2+}$ levels, were significantly increased in heart failure (Fig. 3a). To determine if these changes are compensatory or 
causative, we generated a cardiac-restricted, doxycycline-controlled, mouse model that overexpresses NCLX (NCLX-Tg, Fig. 3b-d). NCLX overexpression enhanced the rate of $\mathrm{Ca}^{2+}$ efflux by $38 \%$ in intact ACMs (Fig. 3e, h), but did not alter ${ }_{\mathrm{m}} \mathrm{Ca}^{2+}$ uptake (Fig. 3f, g), basal $\mathrm{mCa}^{2+}$ content (Fig. 3j, k) or ${ }_{\mathrm{i}} \mathrm{Ca}^{2+}$ cycling (Extended Data Fig. $4 \mathrm{~d}-\mathrm{i}$ ) as compared to aMHC-tetracycline transactivator transgenic (tTA) controls. We found a slight increase in $\Delta \psi$ in NCLX-Tg cardiomyocytes (Extended Data Fig. 4c). The basis for this change is unclear, but it could be simply that NCLX-Tg ACMs were more protected from the stresses of isolation and this result is secondary to improved viability. Monitoring efflux independent of MCU-mediated uptake, with inhibition of sarcoplasmic reticulum and sarcolemmal $\mathrm{Ca}^{2+}$ transport, revealed an $88 \%$ increase in the ${ }_{\mathrm{m}} \mathrm{Ca}^{2+}$ efflux rate (Fig. 3i; Extended Data Fig. 4j). Since we observed a large impact on MPTP opening in NCLX-deleted ACMs, we next examined the $\mathrm{Ca}^{2+}$ load required for $\Delta \psi$ collapse in the overexpression model. NCLX overexpression increased the $\mathrm{Ca}^{2+}$ concentration required for permeability transition by $77 \%$, suggesting enhanced efflux activity was sufficient to suppress MPTP activation (Fig. $31, \mathrm{~m})$. Given the reported role of $\mathrm{mCa}^{2+}$ in the regulation of energetics ${ }^{30}$, we next evaluated oxidative phosphorylation using pyruvate or palmitate as energy substrates. Seahorse analysis of ACMs isolated from NCLX-Tg hearts found no difference in basal respiration, ATP-linked respiration, maximum respiration, spare capacity or proton leak (Extended Data Fig. $4 k-q)$. In agreement with these results, and the finding that there was no change in basal $\mathrm{mCa}^{2+}$ content, we found no baseline change in the NAD:NADH ratio or phosphorylation levels of the $\mathrm{Ca}^{2+}$-responsive PDH complex (Extended Data Fig. 4r, s).

To evaluate if enhanced NCLX activity could limit cell death resulting from ischaemia reperfusion injury we employed in vivo ligation of the left coronary artery for 40 minutes followed by $24 \mathrm{~h}$ of reperfusion (Fig. 4a). Increased NCLX expression reduced infarct size by $43 \%$ (Fig. 4b, c) and the number of TUNEL ${ }^{+}$cells was significantly reduced (Fig. 4d; Extended Data Fig. 5a). NCLX overexpression improved contractile function and reduced superoxide generation after ischaemia reperfusion (Fig. $4 \mathrm{e}-\mathrm{g}$ ).

Next, we subjected NCLX-Tg and tTA controls to permanent occlusion of the left coronary artery to induce a large myocardial infarction and tracked changes in cardiac structure and function for four weeks (Fig. 4h). NCLX-Tg mice displayed less left ventricular dilation and better left ventricular function 2-4 weeks after myocardial infarction (Fig. 4i-k; Extended Data Fig. 5b-e). Overexpression of NCLX also reduced cardiac hypertrophy (Fig. 4l) and the expression of fetal genes associated with the progression of heart failure (Extended Data Fig. 5h, i). Further, NCLX-Tg mice displayed reduced post-infarction fibrosis and inflammation (Fig. 4m, n; Extended Data Fig. 5j-o). Notably, we found a reduction in scar expansion in NCLX-Tg hearts four weeks post-infarction (Fig. 4o), suggesting that NCLX overexpression reduced cell death and salvaged cardiomyocytes at the infarct border zone. We suspect that NCLX overexpression resulted in cardioprotection by increasing mitochondrial efflux capacity, thereby limiting pathogenic $\mathrm{mCa}^{2+}$ accumulation, which we show in this work is linked to a decrease in ROS generation and reduced mitochondrialdependent death signalling. In support of this conclusion, there was a decrease in cardiomyocyte superoxide generation following myocardial infarction (Fig. 4p). 
Our in vivo findings confirm the genetic identity of the mitochondrial $\mathrm{Na}^{+} / \mathrm{Ca}^{2+}$ exchanger and demonstrate that ${ }_{\mathrm{m}} \mathrm{Ca}^{2+}$ efflux is essential for the maintenance of mitochondrial function and cell survival. We conclude that cardiomyocyte $\mathrm{m} \mathrm{Ca}^{2+}$ exchange is active during homeostasis and represents a prominent mitochondrial regulatory mechanism. This is particularly noteworthy, as recent studies employing genetic deletion of MCU have questioned the relevance of basal $\mathrm{m} \mathrm{Ca}^{2+}$ uptake in the heart ${ }^{13-15}$. Furthermore, our results suggest that increasing NCLX expression or activity may be a potential therapeutic route to combat diseases featuring $\mathrm{Ca}^{2+}$ dysregulation.

\section{METHODS}

\section{Generation of SIc8b1 mutant mice}

Embryonic stem (ES) cells were acquired from EUCOMM that were generated by recombinant insertion of a knockout first mutant construct containing lox $P$ sites flanking exons 5-7 of the Slc8b1 gene (chr12: 113298759-113359493) ${ }^{18}$. The ES cell line (clone EPD0460_4_A08) was confirmed by PCR and injected into C57BL/6N blastocysts with subsequent transplantation into pseudo-pregnant females. Chimeric males generated from mutant ES cell injection into albino-B6 blastocysts were selected based on coat colour and subsequently crossbred with C57BL/6N females (Extended Data Fig. 2a). Germline mutant mice were crossbred with ROSA26-FLPe knock-in mice (Jackson Laboratory, stock no. 009086) to remove the FRT-flanked splice acceptor site, $\beta$-gal reporter, and neomycin resistance cassette (Extended Data Fig. 2b). Resultant $S / c 8 b 1^{f l /+}$ mice were interbred to generate homozygous mutant mice with conditional knockout potential $\left(S l c 8 b 1^{f l / f l}\right)$. Next, $S l c 8 b 1^{f l / f l}$ mice were crossed with cardiac-specific-Cre transgenic mice, aMHC-Cre or aMHC-MerCreMre (MCM) (available from Jackson Laboratory, stock no. 005657), to generate cardiomyocyte-specific Slc8b1 knockouts. B6.CMV-Cre transgenic mice (Jackson Laboratory, stock no. 006054) were used for germline deletion. It should be noted that we were unable to generate a germline knockout by either intercrossing the knockout first mutant, in an attempt to make a homozygous gene-trap, or by crossing the $S I c 8 b 1^{f l / f l}$ line to a germline Cre-expressing transgenic mouse, indicating the necessity of $\mathrm{mCa}^{2+}$ extrusion during development. For temporal deletion of Slc8b1 using the MCM model, male mice 812 weeks of age were injected i.p. with tamoxifen ( $40 \mathrm{mg} \mathrm{kg}^{-1}$ per day) for five consecutive days. All mouse genotypes, including controls, received tamoxifen.

\section{Generation of a NCLX cardiac-restricted overexpression mouse model}

We cloned the human SLC8B1 sequence (NM_024959) (5' EcoRI, 3' XmaI) into a plasmid containing the $\mathrm{P}_{\text {tight }}$ Tet-responsive promoter and a SV40 poly(A) sequence and linearized the construct with Xhol digestion followed by gel and Elutip DNA purification. Upon sequence confirmation the purified fragment was injected into the pronucleus of a fertilized ovum and transplanted into pseudo-pregnant females (C57BL/6N). Upon confirmation of germline transmission in founder lines, mutant mice were crossbred with the aMHC-tTA (cardiomyocyte-restricted expression, doxycycline-off) transgenic model. This allowed conditional over-expression upon the withdrawal of chow containing doxycycline (a tetracycline analogue). All mutant mice were fed doxycycline until 4 weeks of age to inhibit embryonic and developmental overexpression. For all experiments, mice were 8-14 weeks 
of age and approved by Temple University's IACUC following all AAALAC guidelines. All mouse experiments were performed in a blinded fashion whenever possible by utilizing a coded ear-tagging system to mask identification by the experimenter.

\section{Western blot analysis}

All protein samples were lysed by homogenization in RIPA buffer. Samples were run by electrophoresis on polyacrylamide Tris-glycine SDS gels. The following antibodies were used in the study: NCLX (1:500, NCKX6 Santa Cruz, sc-161921); MCU (1:1,000, SigmaAldrich, HPA016480); MCUb (1:1,000, Abgent, AP12355b); MICU1 (1:500, custom generation by Yenzyme); EMRE (1:250, Santa Cruz, sc-86337); LETM1 (1:1,000, Proteintech, 16024-1-AP); VDAC (1:2,500, Abcam, ab15895); cyclophilin D (1:5,000, Abcam, ab110324); PDH subunits (1:1,000, Abcam, ab92696), p-PDH ${ }^{\mathrm{S} 293}$ (1:1,000, Abcam, ab110330) ETC respiratory chain complexes (1:5,000, OxPhos Cocktail, Abcam, MS604) and Licor IR secondary antibodies (1:12,000). All blots were imaged on a Licor Odyssey system (anti-mouse, 926-32210; anti-rabbit, 926-68073; anti-goat, 926-32214). Western blot details have been previously reported in detail ${ }^{13}$. All source gels (that is, fulllength western blots) and band density results are available in Supplementary Fig. 1.

\section{Isolation of adult mouse cardiomyocytes (ACMs)}

Cardiomyocytes were isolated from ventricular tissue of adult mice as previously reported ${ }^{13}$.

\section{Adult mouse cardiomyocyte ${ }_{\mathrm{i}} \mathrm{Ca}^{2+}$ and ${ }_{\mathrm{m}} \mathrm{Ca}^{2+}$ transient recordings}

Isolated ACMs were loaded with $1 \mu \mathrm{M}$ Fluo-4 AM (Invitrogen) and placed in a $37{ }^{\circ} \mathrm{C}$ heated chamber on an inverted microscope stage. Cardiomyocytes were perfused with a physiological Tyrode's buffer (150 mM NaCl, $5.4 \mathrm{mM} \mathrm{KCl}, 1.2 \mathrm{mM} \mathrm{MgCl} 2,10 \mathrm{mM}$ glucose, $2 \mathrm{mM}$ sodium pyruvate, and $5 \mathrm{mM}$ HEPES, pH 7.4) containing $2 \mathrm{mM} \mathrm{Ca}^{2+}$. Cells were paced at $0.5 \mathrm{~Hz}$ and ${ }_{\mathrm{i}} \mathrm{Ca}^{2+}$ transients continuously recorded and analysed using Clampex10 software (Molecular Devices). After two minutes of baseline recording, $100 \mathrm{nM}$ isoproterenol (Sigma-Aldrich) was applied by changing the perfusion solution. For intracellular $\mathrm{Ca}^{2+}$ fluorescence measurements, the $F_{0}$ was measured as the average fluorescence of the cell before stimulation. The maximal Fluo-4 fluorescence $(F)$ was measured for peak amplitude. Time to peak was calculated as the time from the beginning of the contraction to peak amplitude. Time to 50\% decay was calculated as the time from the beginning of the contraction to $50 \%$ relaxation. Time to $90 \%$ decay was calculated as the time from the beginning of the contraction to $90 \%$ relaxation. To monitor $\mathrm{mCa}^{2+}$ transients, AAV6-mitycam $\left(1 \times 10^{11}\right.$ particles) was injected intravenously (retro-orbital) into mice. After 2-3 weeks, ACMs were isolated and imaged the same day to monitor $\mathrm{mCa}^{2+}$ flux during pacing $(0.1 \mathrm{~Hz})$. Background fluorescence was subtracted from each experiment before quantification.

\section{Evaluation of $\mathrm{mCa}^{2+}$ uptake and efflux}

To measure ${ }_{\mathrm{m}} \mathrm{Ca}^{2+}$ uptake and efflux, 300,000 ACMs were transferred to intracellular-like medium containing: $120 \mathrm{mM} \mathrm{KCl}, 10 \mathrm{mM} \mathrm{NaCl}, 1 \mathrm{mM} \mathrm{KH}{ }_{2} \mathrm{PO}_{4}, 20 \mathrm{mM}$ HEPES-Tris, 3 $\mathrm{mM}$ thapsigargin, $80 \mu \mathrm{g} \mathrm{ml}^{-1}$ digitonin, protease inhibitors (Sigma EGTA-Free Cocktail), 10 
$\mu \mathrm{M}$ succinate, at $\mathrm{pH}$ 7.2. ACMs were gently stirred and $1 \mu \mathrm{M}$ Fura-FF (Invitrogen) was added to monitor extra-mitochondrial $\mathrm{Ca}^{2+}$. At $20 \mathrm{~s} \mathrm{JC}-1$ (Enzo Life Sciences) was added to monitor $\Delta \psi_{\mathrm{m}}$. Fluorescence signals were monitored at $490 \mathrm{~nm}$ excitation $/ 535 \mathrm{~nm}$ emission for the monomer, $570 \mathrm{~nm}$ excitation/595 nm emission for the J-aggregate of JC-1, and 340 and $380 \mathrm{~nm}$ excitation/510 nm emission for Fura-FF to calculate ratiometric changes. At 350 s a $20 \mu \mathrm{M} \mathrm{Ca}^{2+}$ bolus was added. Clearance of extra-mitochondrial $\mathrm{Ca}^{2+}$ was representative of $\mathrm{mCa}^{2+}$ uptake. At $550 \mathrm{~s}, 1 \mu \mathrm{M}$ Ru360 (MCU-inhibitor) was added to inhibit uptake and allow for quantification of $\mathrm{m}_{\mathrm{m}} \mathrm{Ca}^{2+}$ efflux. At $650 \mathrm{~s}, 10 \mu \mathrm{M}$ CGP-37157 (NCLX inhibitor) was added to block $\mathrm{mCa}^{2+}$ efflux. At completion of the experiment the protonophore, FCCP, was added. All experiments were conducted at $37{ }^{\circ} \mathrm{C}$ and recorded on a PTI spectrofluorometer. All details have been previously reported ${ }^{13}$.

To measure $\mathrm{m}_{\mathrm{Ca}}{ }^{2+}$ uptake capacity, 300,000 adult cardiomyocytes were gently stirred and 1 $\mu \mathrm{M}$ Fura-FF (Invitrogen) was added to monitor extra-mitochondrial $\mathrm{Ca}^{2+}$. Fura was monitored at 340/380 nm excitation and $510 \mathrm{~nm}$ emission. Starting at $350 \mathrm{~s} 10 \mu \mathrm{M} \mathrm{Ca}^{2+}$ boluses were added every $60 \mathrm{~s}$. Clearance of extra-mitochondrial $\mathrm{Ca}^{2+}$ was representative of $\mathrm{mCa}^{2+}$ uptake. At completion of the experiment the protonophore, FCCP, was added. All experiments were conducted in ${ }_{\mathrm{i}} \mathrm{Ca}^{2+}$ buffer containing $\mathrm{Na}^{+}$at $37{ }^{\circ} \mathrm{C}$ and recorded on a PTI spectrofluorometer. All details have been previously reported ${ }^{13}$.

\section{${ }_{m} \mathrm{Ca}^{2+}$ flux and uptake capacity in NCLX kncokdown HeLa Cells}

HeLa cells (ATCC) were transduced with lentivirus encoding shRNA targeting SLC8B1 (Mission shRNA, Sigma).

\section{TRCN0000005045 (CCGGGCCTTCTTGCTGTCATGCAATCTCGAGATTGC ATGACAGCAAGAAGGCTTTTT) \\ TRCN0000005046 (CCGGCCGGGTATCTTCTAATACCAACTCGAGTTGGT ATTAGAAGATACCCGGTTTTT) \\ TRCN0000005047 (CCGGCCTCACTGAATTTGGAGTGATCTCGAGATCAC TCCAAATTCAGTGAGGTTTTT) \\ TRCN0000005048 (CCGGGTGTGCTTTGTGTGCTGCTAACTCGAGTTAGC AGCACACAAAGCACACTTTTT)}

Cells were selected with puromycin and gene expression was evaluated by qPCR. The two cell lines with the lowest SLC8B1 expression, sh5045 (shRNA1) and sh5048 (shRNA2), were used in subsequent experiments. Cells were infected with AAV-mitycam to measure ${ }_{\mathrm{m}} \mathrm{Ca}^{2+}$ flux. Cells were imaged in Tyrode's buffer $(150 \mathrm{mM} \mathrm{NaCl}, 5.4 \mathrm{mM} \mathrm{KCl}, 5$ $\mathrm{mM}$ HEPES, $10 \mathrm{mM}$ glucose, $2 \mathrm{mM} \mathrm{CaCl}_{2}, 2 \mathrm{mM}$ sodium pyruvate at $\mathrm{pH}$ 7.4) on a Zeiss 510 confocal microscope using the $488 \mathrm{~nm}$ laser. $\mathrm{Ca}^{2+}$ flux was assessed in real-time, collecting data every $3 \mathrm{~s}$ and analysed on Zen software. $\mathrm{mCa}^{2+}$ uptake capacity in nonexcitable cells was evaluated by permeabilizing $4 \times 10^{6}$ cells in the intracellular-like medium detailed above. Extra-mitochondrial $\mathrm{Ca}^{2+}$ was monitored with Fura-FF and JC-1 was added to monitor $\Delta \psi$ as described above. At $500 \mathrm{~s}$, a $5 \mu \mathrm{M}$ pulse of $\mathrm{Ca}^{2+}$ was added then sequentially added every $100 \mathrm{~s}$ ( 5 additions). FCCP was added at the end of each experiment. 


\section{Echocardiography}

Transthoracic echocardiography of the left ventricle was performed and analysed on a Vevo 2100 imaging system (VisualSonics) as previously reported ${ }^{13}$. Mice were anaesthetized with $2 \%$ isoflurane in $100 \%$ oxygen during acquisition. B-mode and M-mode images were collected in long- and short-axis. M-mode axis and B-mode strain analysis were performed using VisualSonics software for both short- and long-axis images.

\section{Histology, tissue gravimetrics, image analysis}

For histological analysis, hearts were collected at the indicated time points and fixed in 10\% buffered formalin. Next, hearts were dehydrated and embedded in paraffin. Serial 5- $\mu \mathrm{m}$ sections were cut and stained with Masson's trichrome to analyse fibrosis and H\&E stain to examine inflammation. Cardiomyocytes cross-sectional area (CSA) was analysed by staining the heart sections with a wheat germ agglutinin-FITC conjugate at $100 \mu \mathrm{g} \mathrm{m}{ }^{-1}$ to highlight sarcolemma (minimum of $n=80$ cardiomyocytes measured per heart). ImageJ was used to quantify all histological endpoints. For evaluating lung oedema, the lungs were weighed at the time of isolation and after dehydration at $37{ }^{\circ} \mathrm{C}$ for 1 week.

\section{Transmission electron microscopy}

Left ventricular samples were fixed with $2 \%$ glutaraldehyde and $2 \%$ paraformaldehyde in $0.1 \mathrm{M}$ sodium cacodylate buffer $\mathrm{pH} 7.4$ containing $2 \mathrm{mM}$ calcium chloride and cut into 2$\mathrm{mm}^{3}$ pieces. The tissues were washed with $0.1 \mathrm{M}$ sodium cacodylate buffer $\mathrm{pH} 7.4$ containing $2 \mathrm{mM}$ calcium chloride and then fixed with freshly prepared $1 \%$ osmium tetroxide and $1.5 \%$ potassium ferrozyanide in $0.1 \mathrm{M}$ sodium cacodylate buffer $\mathrm{pH} 7.4$ containing $2 \mathrm{mM}$ calcium chloride for $1 \mathrm{~h}$ on ice. Following buffer washes, the samples were fixed for an additional $1 \mathrm{~h}$ at room temperature with $2 \%$ osmium tetroxide (aq). The tissues were washed with water and then en bloc stained with $2 \%$ uranyl acetate (aq) for $2 \mathrm{~h}$. After additional washes with water, samples were dehydrated in an ascending acetone series (25\%, $50 \%, 75 \%, 95 \%, 100 \%, 100 \% ; 15$ minutes each), transitioned into propylene oxide, and infiltrated with Embed-812 resin over a period of $48 \mathrm{~h}$. The tissue was embedded and polymerized at $60^{\circ} \mathrm{C}$ for $24-48 \mathrm{~h}$. Samples (three blocks per mouse) were sectioned on a Reichert-Jung Ultracut E ultramicrotome, and sections were collected onto 200 mesh formvar-carbon coated copper grids and post-stained with $2 \%$ uranyl acetate in 50\% methanol and Reynolds lead citrate ${ }^{31}$. Sections were examined with a Zeiss Libra 120 transmission electron microscope operating at $120 \mathrm{kV}$, and images were acquired with a Gatan Ultrascan 1000 CCD. Cristae density was calculated using ImageJ planimetry calibrated for area void of cristae and mitochondrial size was calculated by tracing individual mitochondria after calibration for distance (minimum of 200 mitochondria analysed per mouse, $n=3-4$ mice per group).

\section{ECG recording}

Mice were surgically implanted with pseudo-1 lead ETA-F20 transmitters (DSI) as previously reported ${ }^{32}$. Continuous ECG recordings were monitored and analysed using Chart 7 Pro. 


\section{Mitochondria isolation and swelling assays}

Hearts were extracted from mice and mitochondria were isolated using a published protocol $^{33}$. For the swelling assay, mitochondria were diluted in mitochondrial assay buffer containing (125 mM KCl, $20 \mathrm{mM}$ HEPES, $2 \mathrm{mM} \mathrm{MgCl}_{2}, 10 \mathrm{mM} \mathrm{NaCl}, 2 \mathrm{mM} \mathrm{K}_{2} \mathrm{HPO}_{4}$ at $\mathrm{pH}$ 7.2) and supplemented with $10 \mathrm{mM}$ succinate. Swelling was assessed using a Tecan Infinite M1000 Pro plate reader measuring absorbance at $540 \pm 10 \mathrm{~nm}$ every $5 \mathrm{~s}$. A $500 \mu \mathrm{M}$ $\mathrm{CaCl}_{2}$ pulse was added to induce mitochondrial swelling (that is, decrease in absorbance). Mitochondrial shrinkage was induced by altering the buffer osmolality by addition of $5 \%$ polyethylene glycol 3350 (PEG).

\section{Evaluation of reactive oxygen species generation}

Live myocardium was isolated from mice and heart sections were sliced using a McIlwain Tissue Chopper. Tissue was stained with $20 \mu \mathrm{M}$ dihydroethidium (DHE) for 30 minutes at $37^{\circ} \mathrm{C}$ and imaged on a Zeiss Axio Observer Z1 fluorescent microscope at $490 \pm 10 \mathrm{~nm}$ excitation and $632 \pm 30 \mathrm{~nm}$ emission. Oxidized DHE fluoresces red and intercalates DNA. To measure mitochondrial superoxide production isolated ACMs were loaded with $5 \mu \mathrm{M}$ MitoSOX Red for 10 minutes at $37^{\circ} \mathrm{C}$, washed and imaged at $490 \pm 10 \mathrm{~nm}$ excitation and $585 \pm 20 \mathrm{~nm}$ emission.

\section{Evans blue dye (EBD) exclusion assay ${ }^{34}$}

To delineate cells with ruptured/compromised membranes, mice were injected with $250 \mu \mathrm{l}$ of $1 \%$ EBD i.p. $18 \mathrm{~h}$ before death. Hearts were isolated and fixed in OCT and cryosectioned at $5 \mu \mathrm{m}$ and co-stained with wheat-germ agglutinin as described above. Images were acquired on a Zeiss Axio Observer Z1 fluorescent microscope at 490/20 nm excitation and 535/50 nm emission for WGA and $575 \pm 17.5 \mathrm{~nm}$ excitation and $632 \pm 30 \mathrm{~nm}$ emission for EBDpositive cardiomyocytes.

\section{Human left ventricular tissue samples}

Human myocardium was obtained from end-stage failing hearts explanted at time of cardiac transplantation. Non-failing donor hearts that were deemed unsuitable for transplantation were used as control. Prior to explant, hearts underwent intra-operative antegrade coronary perfusion with 4:1 blood cardioplegia solution. Following arrest, hearts were explanted and placed into cold $\mathrm{Ca}^{2+}$-free, modified Krebs-Henseleit solution as previously described ${ }^{35}$. Samples were taken from mid-myocardial regions of the left ventricular free wall in areas void of scar tissue. All tissue samples were rapidly frozen in liquid nitrogen and stored at $-80^{\circ} \mathrm{C}$ until isolation of RNA and/or protein. Prospective written informed consent for research use of heart tissue was obtained from all transplant recipients or their next of kin (non-failing donors). Non-failing hearts were unsuitable for transplantation due to donor age over 60 years, coronary artery disease without infarction, or positive hepatitis serology. Patient consent, sample collection and preparation, and clinical data collection were all performed according to a protocol approved by the Lewis Katz School of Medicine Institutional Review Board. 


\section{qPCR mRNA analysis}

mRNA was isolated using the Qiagen RNeasy Kit. qPCR analysis was conducted following manufacturer instructions (Maxima SYBR, Thermo Scientific). We evaluated samples for mRNA expression of Slc8b1, Mcu, Micu1, Nppa, Nppb, Spp1, Postn1, Acta2, II1b and II6 mRNA expression. Rps13 was used as a housekeeping gene. All experiments samples were analysed in triplicate and averaged. To calculate fold change in mRNA expression, the $2^{-\Delta \Delta C_{\mathrm{t}}}$ method was used.

\section{Myocardial ischaemia reperfusion injury and infarction}

LCA ligation was performed as previously described in ref. 36. In brief, mice were anaesthetized with isoflurane and the heart exposed via a left thoracotomy at the fifth intercostal space. The left coronary artery (LCA) was permanently ligated to induce a large myocardial infarction. Alternatively, for ischaemia reperfusion, a slipknot was tied around the LCA to enable reperfusion. The heart was returned to the chest cavity and the wound was sutured revealing the slipknot. After 40 minutes of ischaemia, the slipknot was released and the ischaemic area was allowed to reperfuse for $24 \mathrm{~h}$. To assess infarct size, after religation of the LCA, hearts were injected with $3 \%$ Evans blue to delineate the area not at risk and 1-mm heart sections were cut using a McIlwain Tissue Chopper. Heart sections were incubated with $1 \%$ triphenyl tetrazolium chloride (TTC) for $5 \mathrm{~min}$ at $37{ }^{\circ} \mathrm{C}$ to demarcate viable tissue. Each slice was weighed and ImageJ was used to quantify infarct area as previously reported ${ }^{13}$.

\section{Metabolic assays}

A Seahorse Bioscience XF96 extracellular flux analyser was employed to measure adult cardiomyocyte oxygen consumption rates (OCR). About 2,500 cardiomyocytes per well were plated in XF media pH 7.4 supplemented with $25 \mathrm{mM}$ glucose and $1 \mathrm{mM}$ sodium pyruvate or $0.1 \mathrm{mM}$ palmitate. Basal OCR was measured, then $3 \mu \mathrm{M}$ oligomycin was injected to inhibit ATP-linked respiration, followed by $1.5 \mu \mathrm{M}$ FCCP to measure maximal respiration, and finally to completely inhibit all mitochondrial respiration, $2 \mu \mathrm{M}$ rotenone/ antimycin-A was injected. Detailed methodology has been previously reported ${ }^{13}$.

\section{Invasive haemodynamic measurements}

Mice were anaesthetized (avertin, $25 \mathrm{mg} \mathrm{kg}^{-1}$ ) and a cervical incision was performed to isolate the right carotid artery for insertion of a 1.4-F pressure catheter (SPR-671, Millar Instruments). The pressure transducer was advanced into the left ventricle before recording. All data was analysed using Chart 6.0 software.

\section{Statistics}

All results are presented as mean \pm s.e.m. All experiments were replicated at least three times if biological replicates were not appropriate. Statistical powering was initially performed using the nQuery Advisor 3.0 software (Statistical Solutions) along with historical data to estimate sample size. For all experiments, the calculations use $a=0.05$ and $\beta=0.2$ (power= 0.80). Statistical analysis was performed using Prism 6.0 (Graph Pad Software). Where appropriate, column analyses were performed using an unpaired, two- 
tailed $t$-test (for two groups) or one-way ANOVA with Tukey correction (for groups of three or more). For grouped analyses either multiple unpaired $t$-tests with correction for multiple comparisons using the Holm-Sidak method were used or, where appropriate, two-way ANOVA with a Sidak post hoc or Tukey post hoc analysis was performed. $P$ values less than 0.05 (95\% confidence interval) were considered significant. All data displayed a normal distribution and variance was similar between groups for each evaluation. For all in vivo studies, researchers were blinded from mouse genotypes and a numerical ear tagging system enabled unbiased data collection. Mice were excluded from the myocardial infarction study if they lacked a scar or infarct, as evaluated by histological staining 4 weeks after ligation.

\section{Data availability}

All relevant data are available from the authors.

\section{Extended Data}
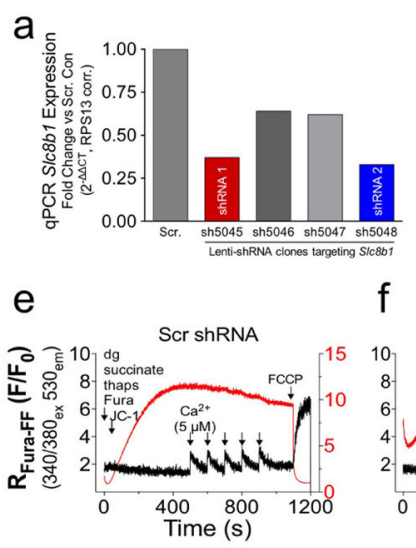

b

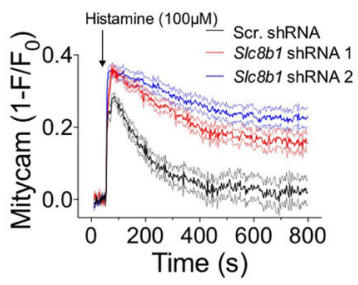

$\mathrm{f}$
SIC8b1 ShRNA 1

g
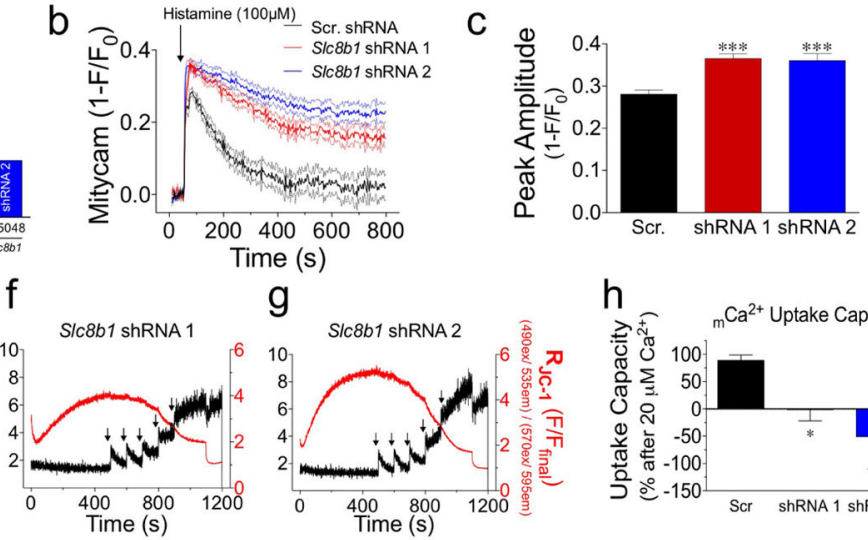

d
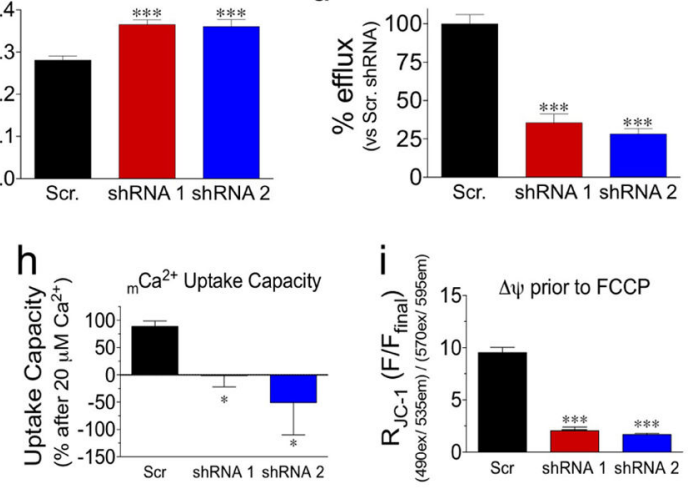

Extended Data Figure 1. shRNA knockdown of NCLX and biophysical characterization in nonexcitable cells

a, qPCR analysis of $S L C 8 B 1 \mathrm{mRNA}$ expression in shRNA stable knockdown HeLa cell lines versus scramble shRNA control cell line (scr. con). $\mathbf{b}, \mathrm{m}^{2+}$ transients recorded in cells expressing the genetic $\mathrm{m}^{2+}$ sensor, mitycam, during histamine treatment $(100 \mu \mathrm{M})$. Mean intensity shown as solid lines, thin dashed lines display s.e.m. c, $\mathrm{mCa}^{2+}$ peak amplitude. d, Per cent ${ }_{\mathrm{m}} \mathrm{Ca}^{2+}$ efflux versus scramble shRNA control. $n=42-49$ cells per group. e-g, HeLa cells were loaded with the $\mathrm{Ca}^{2+}$ sensor, Fura-FF, and the $\Delta \psi$ sensor, JC-1, permeabilized with digitonin (dg) and treated with SERCA inhibitor, thapsigargin (thaps) for simultaneous ratiometric monitoring during repetitive additions of $5 \mu \mathrm{M} \mathrm{Ca}^{2+}$ (black arrows). FCCP was used to collapse $\Delta \psi$ at the conclusion of each experiment. $\mathbf{h}, \mathrm{mCa}^{2+}$ uptake capacity versus scramble shRNA control cells following $20 \mu \mathrm{M} \mathrm{Ca}^{2+}$ (fourth pulse). i, JC-1-derived $\Delta \psi$ before FCCP addition. $n=3$ experiments per assay; $* P<0.05$, *** $P<$ 0.001 versus scramble shRNA control. 
a
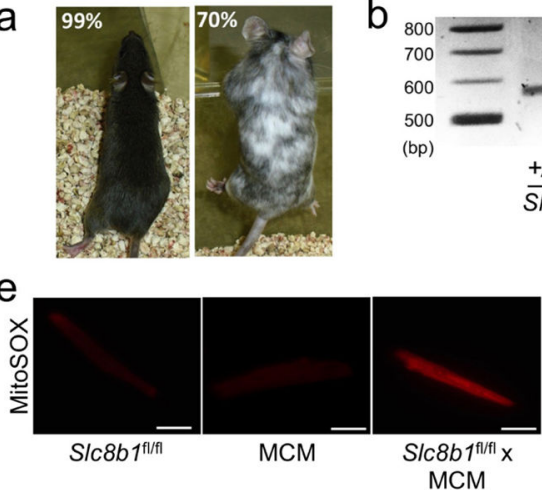

$\mathrm{h}$

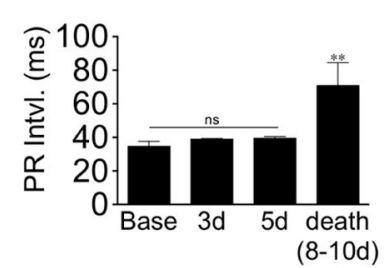

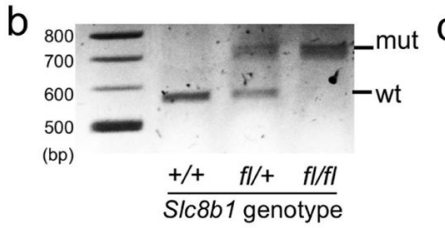

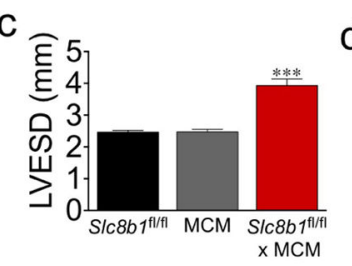

d

Curve Fitting with $95 \%$ Confidence Intervals
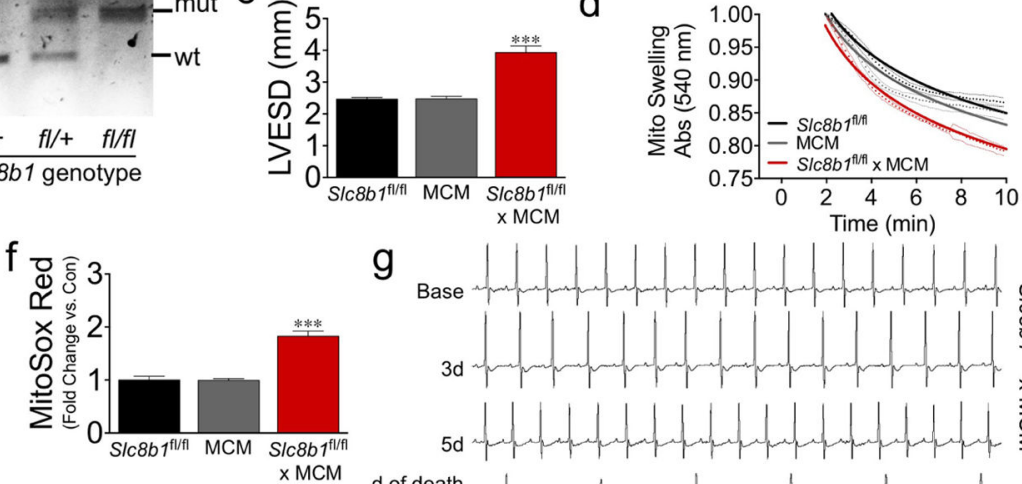

g

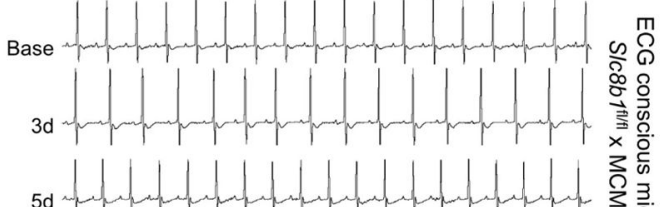

$(8 d-10 d)$
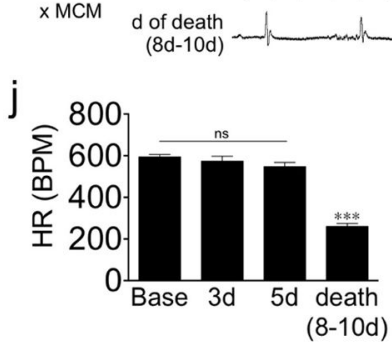

$\mathrm{k}$

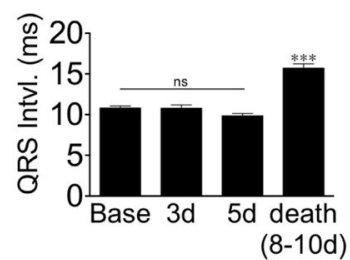

$\mathrm{m}$

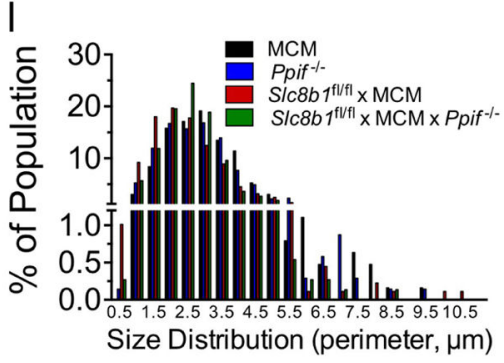

$\mathrm{n}$
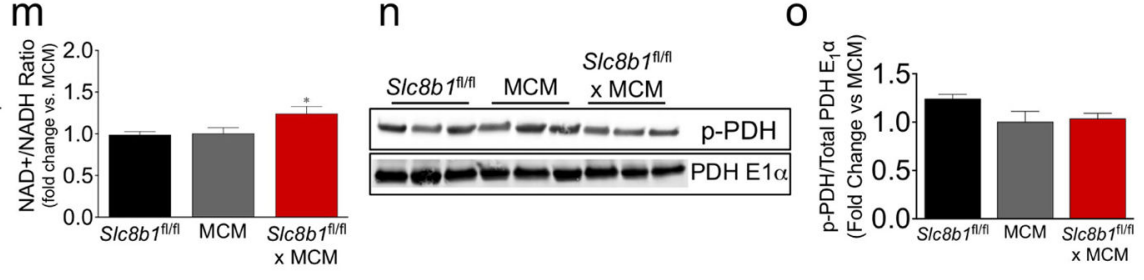

Extended Data Figure 2. Characterization of Slc8b1 conditional knockout following tamoxifen administration

a, Images of chimeric founder mice and estimated per cent chimerism, black coat colour correlates with mutant ES cell contribution to development. b, Image of gel with PCR genotyping results for $S 1 c 8 b 1$ lox $P$ targeted mice. c, Left ventricular end-systolic dimension (LVESD) three days after tamoxifen administration. d, Curve fitting of mitochondrial swelling traces after addition of $500 \mu \mathrm{M} \mathrm{Ca}^{2+}$. e, Representative images of MitoSOX Redstained ACMs (scale bar, $40 \mu \mathrm{m}$ ). f, Fold change in MitoSOX Red intensity three days after tamoxifen administration. $n=42-52$ ACMs per group. g, Representative ECG recordings of $S l c 8 b 1^{f l / f l} \times$ MCM mice $(n=4-7)$ at baseline, three days and five days after tamoxifen treatment, and day of sinus arrest. h, Quantitation of PR interval. i, Quantitation of QRS interval. j, Quantitation of heart rate (HR). BPM, beats per minute. k, Western blot of proposed MPTP components from heart protein lysate: CypD and ANT, VDAC shown in Fig. 1d. l, Size distribution (perimeter) of mitochondria analysed in electron microscopy images three days after tamoxifen (minimum 200 mitochondria quantified per mouse, $n=3-$ 4 mice per group). $\mathbf{m}$, Quantification of $\mathrm{NAD}^{+} / \mathrm{NADH}$ ratio three days after tamoxifen administration (fold change versus control). $n=6-7$ per group. $\mathbf{n}$, Western blots of total pyruvate dehydrogenase (PDH E1a) and phosphorylated-PDH (p-PDH) from heart protein 
lysate. o, Fold change in the ratio of p-PDH/total PDH E1a versus control. $n=3$ per group;

$* P<0.05, * * P<0.01, * * * P<0.001 ;$ ns, not significant.

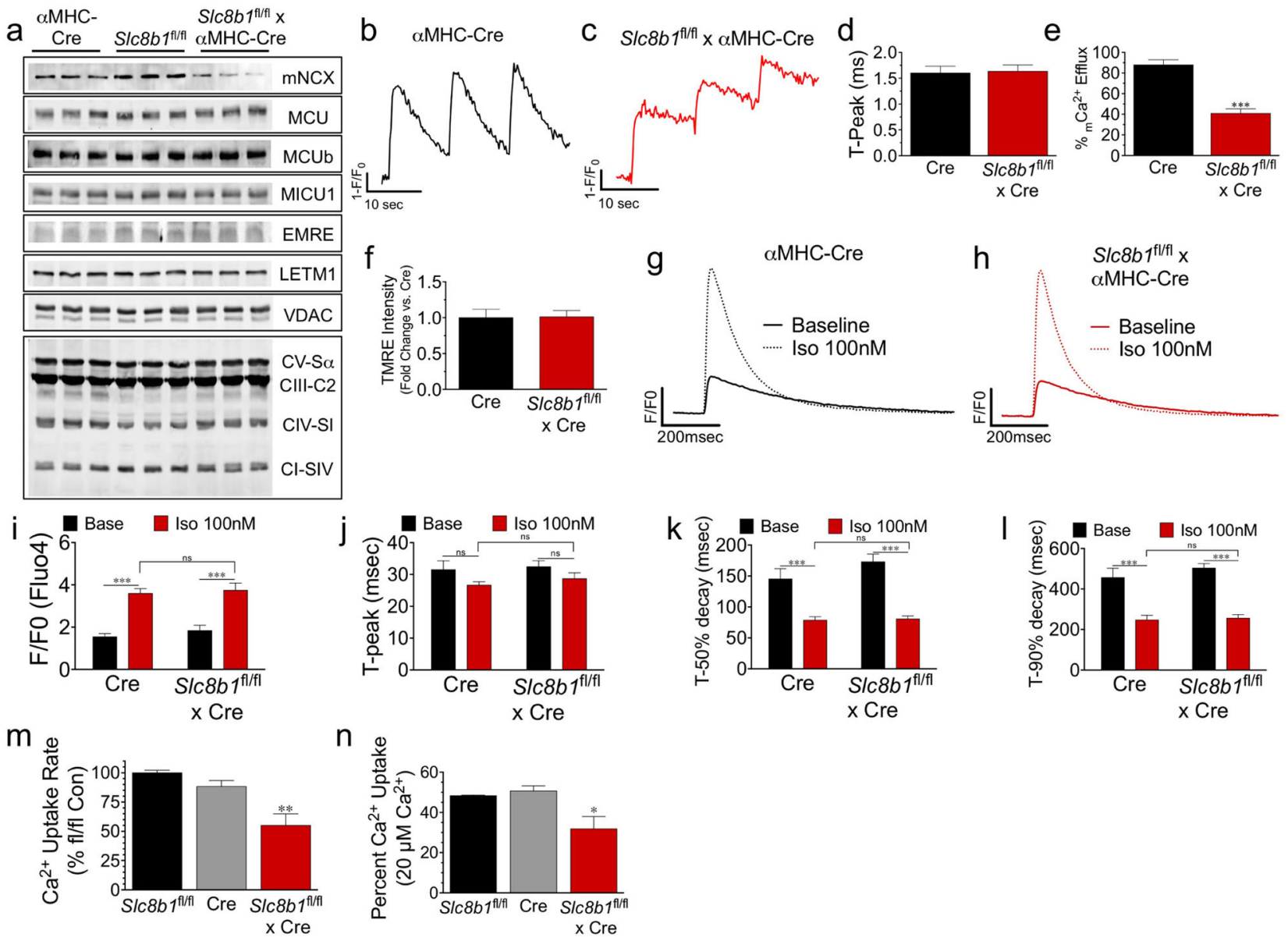

Extended Data Figure 3. Examination of $\mathrm{Ca}^{2+}$ flux following aMHC-Cre-mediated deletion of Slc8b1.

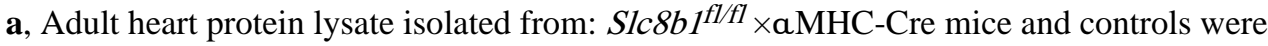
examined by western blot for NCLX expression and various other proteins involved in $\mathrm{Ca}^{2+}$ exchange: MCU, MCUb, MICU1, EMRE, LETM1, VDAC; and OXPHOS components: ATP synthase subunit a (CV-Sa), complex III subunit core 2 (CIII-C2), complex IV subunit I (CIV-SI), and complex I subunit NDUFB8 (CI-SIV). $n=3$ hearts per group. b, c, Series of mitycam $\mathrm{mCa}^{2+}$ transients recorded during pacing $(0.1 \mathrm{~Hz}) . \mathbf{d}, \mathrm{m}^{2+}$ time to peak amplitude. e, Per cent ${ }_{\mathrm{m}} \mathrm{Ca}^{2+}$ efflux $10 \mathrm{~s}$ after pacing. f, Tetramethylrhodamine, ethyl ester (TMRE) $(\Delta \psi)$ in intact ACM. $n=23-24$ ACMs per group. $\mathbf{g}, \mathbf{h}$, Representative recordings of ${ }_{\mathrm{i}} \mathrm{Ca}^{2+}$ transients (Fluo-4) in ACMs paced at $1 \mathrm{~Hz}$ with or without isoproterenol (Iso, $100 \mathrm{nM}$ ). $\mathbf{i},{ }_{\mathrm{i}} \mathrm{Ca}^{2+}$ peak amplitude. $\mathbf{j}$, ${ }_{\mathrm{i}} \mathrm{Ca}^{2+}$ time to peak amplitude. $\mathbf{k}, \mathrm{Ca}^{2+}$ time to $50 \%$ decay. l, Time to $90 \%$ decay. $n=10$ cells per group. $\mathbf{m}, \mathbf{n}$, Permeabilized $\mathrm{ACM}_{\mathrm{m}} \mathrm{Ca}^{2+}$ uptake rate $(\mathbf{m})$ and per cent uptake $(\mathbf{n})$ after $\mathrm{Ca}^{2+}$ addition. $* P<0.05$, $* * P<0.01, * * * P$ $<0.001$. 

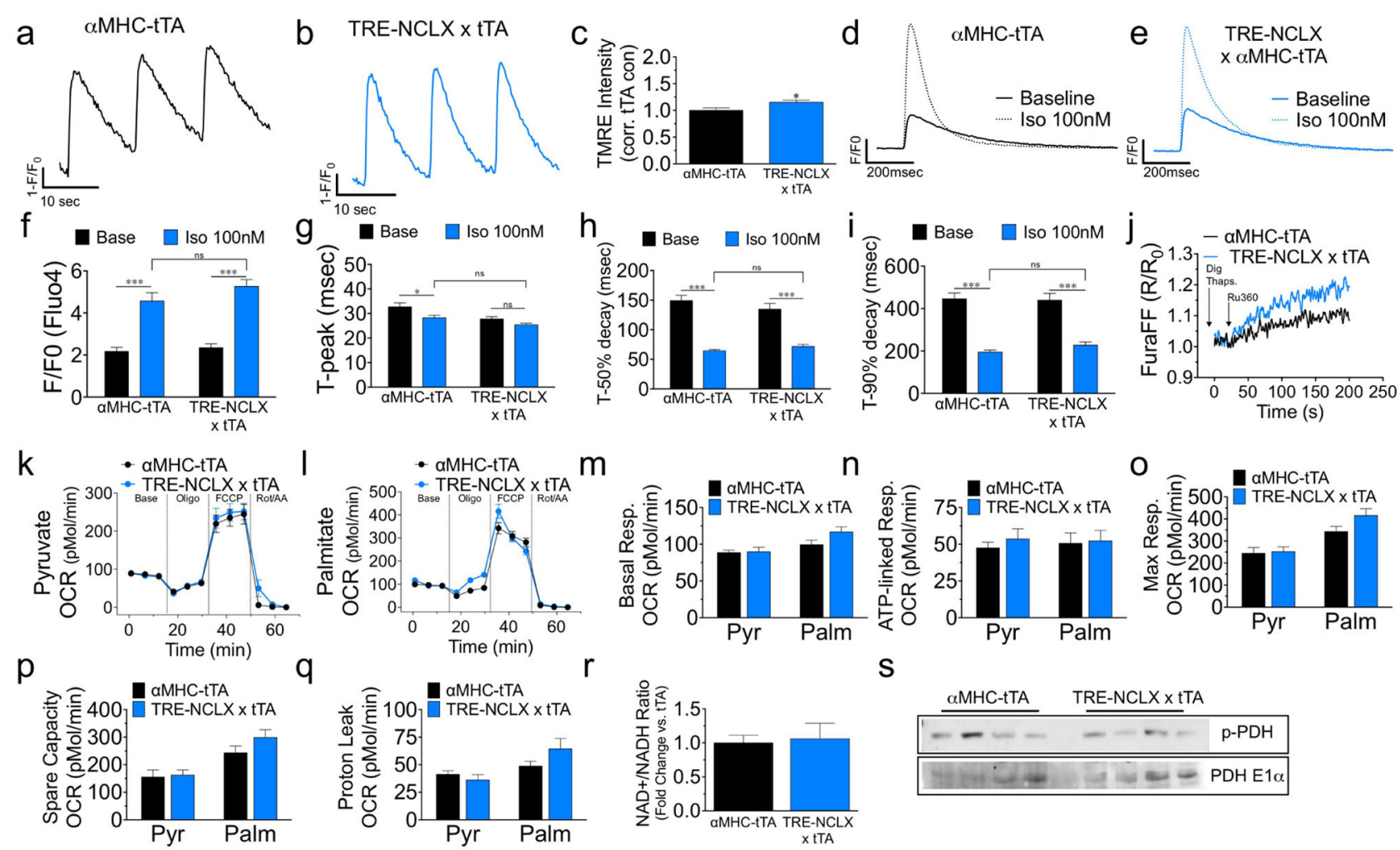

Extended Data Figure 4. Characterization of NCLX transgenic mice $\mathbf{a}, \mathbf{b}$, Series of mitycam $\mathrm{maa}^{2+}$ transients recorded during pacing $(0.1 \mathrm{~Hz}) . \mathbf{c}$, Tetramethylrhodamine, ethyl ester (TMRE) $(\Delta \psi)$ in intact ACMs. $n=23-36$ ACMs per group. d, e, Representative traces of ${ }_{\mathrm{i}} \mathrm{Ca}^{2+}$ transients (Fluo-4) in ACMs paced at $1 \mathrm{~Hz}$ with or without isoproterenol $(100 \mathrm{nM}) . \mathbf{f},{ }_{i} \mathrm{Ca}^{2+}$ peak amplitude. $\mathbf{g},{ }_{\mathrm{i}} \mathrm{Ca}^{2+}$ time to peak amplitude. $\mathbf{h}$, Time to $50 \%$ decay. i, Time to $90 \%$ decay ( $n=15$ cells per group). $\mathbf{j}$, Recording of transients in Digitonin (dig)-permeabilized ACMs loaded with Fura-FF, treated with thapsigargin (thaps) and placed in $20 \mu \mathrm{M}$ bath $\mathrm{Ca}^{2+}$ before treatment with the $\mathrm{MCU}$ inhibitor, Ru360, to quantify the rate of ${ }_{\mathrm{m}} \mathrm{Ca}^{2+}$ efflux independent of uptake. $\mathbf{k}-\mathbf{q}$, Seahorse analysis of mitochondrial oxygen consumption rates (OCR) in ACMs in the presence of pyruvate or palmitate. $\mathbf{m}$, Basal OCR. $\mathbf{n}$, ATP-linked respiration after addition of ATP synthase inhibitor, oligomycin. o, Maximal respiration after addition of protonophore, FCCP. p, Spare respiratory capacity (max - basal). q, Proton leak (post-oligomycin OCR non-mitochondrial OCR). $n=11-15$ per condition. $\mathbf{r}$, Quantification of $\mathrm{NAD}^{+} / \mathrm{NADH}$ ratio three days after tamoxifen (fold change versus control). $n=4-5$ per group. $\mathbf{s}$, Western blot of total pyruvate dehydrogenase (PDH E1a) and phosphorylated-PDH (p-PDH) from heart protein lysate. $* P<0.05, * * * P<0.001$. 

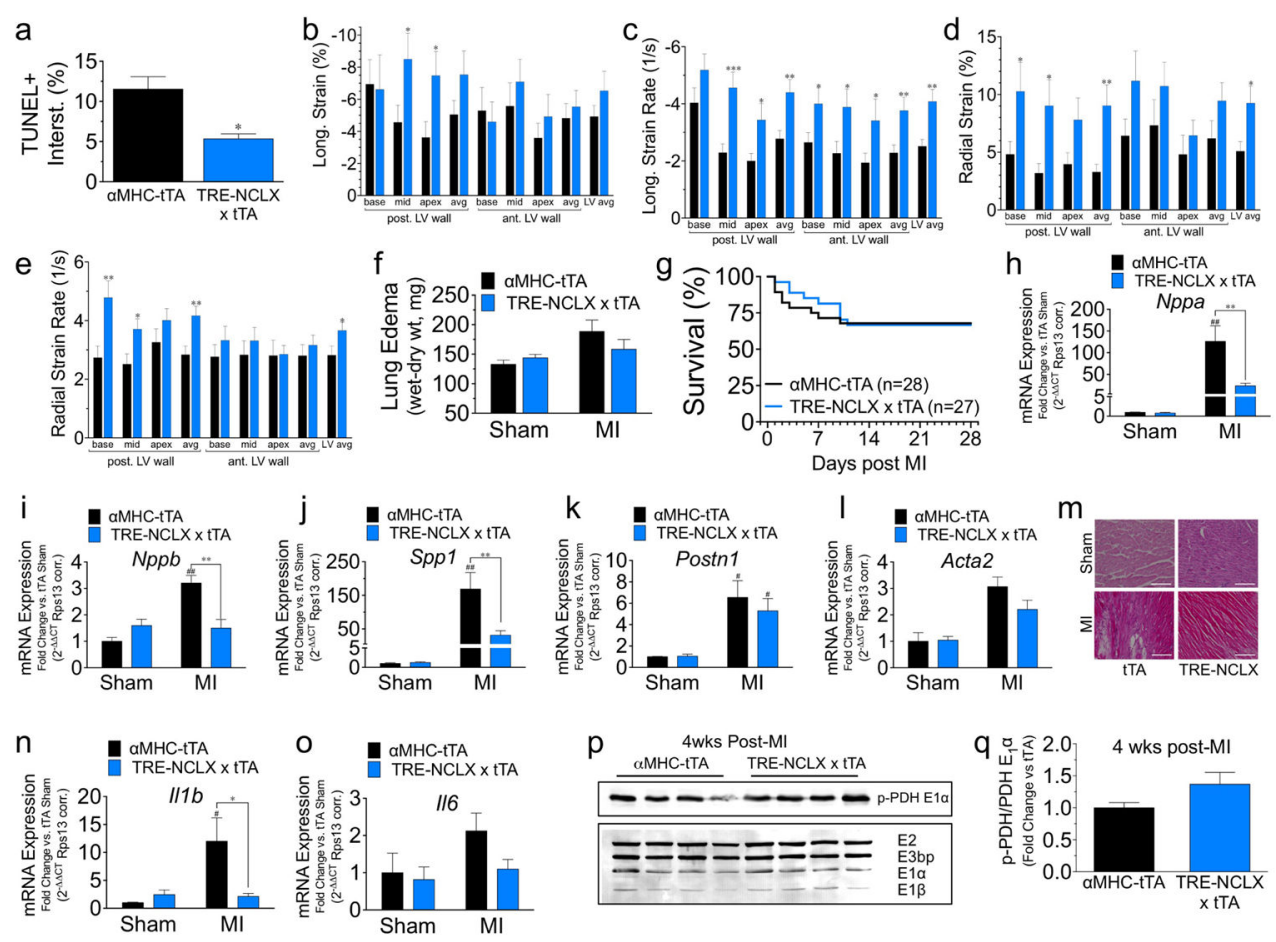

Extended Data Figure 5. Analysis of NCLX-Tg mice $24 \mathrm{~h}$ after ichaemia reperfusion and 4 weeks after myocardial infarction

a, Quantification of TUNEL ${ }^{+}$interstitial cells after ischaemia reperfusion. b-e, B-mode speckle tracking analysis of left ventricular function 4 weeks after myocardial infarction: longitudinal strain (b), longitudinal strain rate (c), radial strain $(\mathbf{d})$, radial strain rate $(\mathbf{e}) . n=$ 20 tTA and $n=18$ NCLX-Tg. f, Lung oedema (wet-dry lung weight). $n=7$ per sham group, $n=17$ per myocardial infarction group. $\mathbf{g}$, Kaplan-Meier survival curves post myocardial infarction. $n=28$ tTA and $n=27$ NCLX-Tg. $\mathbf{h}-\mathbf{l}$, qPCR quantification of mRNA expression in hearts from sham-treated mice or 4 weeks after myocardial infarction. Nppa, atrial natriuretic peptide; $N p p b$, brain natriuretic peptide; Spp1, osteopontin; Postn1, periostin; Acta2, smooth muscle a-actin (sham, $n=3$ group; myocardial infarction, $n=7$ per group). $\mathbf{m}$, Representative images of H\&E-stained heart sections from the infarct border zone 4 weeks post myocardial infarction. Scale bar, $400 \mu \mathrm{m}$. $\mathbf{n}, \mathbf{o}$, qPCR analysis of mRNA expression for inflammatory cytokines 4 weeks post myocardial infarction. II1b, interleukin-1 $\beta$; Il6, interleukin-6. $n=3$ per sham group, $n=7$ per myocardial infarction group. p, Western blot of pyruvate dehydrogenase subunits and phosphorylated-PDH (pPDH) from heart protein lysate 4 weeks post myocardial infarction. q, Fold-change in the ratio of p-PDH/total PDH E1a versus tTA control. $n=4$ per group; $* P<0.05$, $* * P<0.01$, $* * * P<0.001$, versus a MHC tTA post injury; $\# P<0.05$, \#\# $P<0.01$ versus sham control. 


\section{Extended Data Table 1}

Electrocardiography measurements of $S I c 8 b 1^{f l / f l} \times \operatorname{MCM}$ mice $(n=7)$ before and after the delivery of tamoxifen

\begin{tabular}{|c|c|c|c|c|c|c|c|c|c|}
\hline $\begin{array}{c}\text { Time } \\
\text { (post-tamox) }\end{array}$ & RR Int. (ms) & Heart Rate (BPM) & PR int. (ms) & P Dur. (ms) & QRS Int. (ms) & QT Int. (ms) & QTe (ms) & JT Int. (ms) & $\begin{array}{l}\text { T-peak-T- } \\
\text { end (ms) }\end{array}$ \\
\hline Base & $101.1 \pm 1.9$ & $594.6 \pm 11.3$ & $34.6 \pm 3.1$ & $12.3 \pm 1.1$ & $10.9 \pm 0.2$ & $18.3 \pm 1.1$ & $57.2 \pm 3.0$ & $7.4 \pm 1.1$ & $4.9 \pm 1.0$ \\
\hline 3d & $105.7 \pm 4.7$ & $573.6 \pm 24.2$ & $39.0 \pm 0.4$ & $14.6 \pm 1.0$ & $10.9 \pm 0.4$ & $17.9 \pm 0.6$ & $55.1 \pm 2.3$ & $7.1 \pm 0.9$ & $4.5 \pm 0.8$ \\
\hline 5d & $111.2 \pm 3.9$ & $547.8 \pm 20.1$ & $39.7 \pm 0.9$ & $11.7 \pm 1.5$ & $10.0 \pm 0.3$ & $16.7 \pm 0.7$ & $50.1 \pm 2.7$ & $6.9 \pm 0.7$ & $4.3 \pm 0.6$ \\
\hline Death (8d-10d) & $243.1 \pm 12.4^{*}$ & $255.6 \pm 12.7 *$ & $62.8 \pm 13.8^{*}$ & $14.5 \pm 1.4$ & $16.0 \pm 0.4 *$ & $28.4 \pm 1.7^{*}$ & $57.8 \pm 3.5$ & $12.5 \pm 1.5$ & $7.8 \pm 1.1$ \\
\hline
\end{tabular}

Measurements include: RR interval (time between successive R waves); heart rate (beats per minute); PR interval (time between P and R waves); P duration (time from start to end of P wave); QRS interval (duration of start of Q wave to end of $\mathrm{S}$ wave); QT interval (start of Q to end of T wave); QT interval corrected to heart rate; JT interval (time between J and T waves); $T$ wave time from peak to end. Death indicates recordings acquired on the day of each death (8-10 days after tamoxifen administration).

${ }^{*} P<0.05$.

\section{Extended Data Table 2}

Genotype distribution of $S / c 8 b 1 \times$ a MHC-Cre mice

\begin{tabular}{|c|c|c|}
\hline Genotype & Observed \# $(\%$ total $)$ & Expected \# $(\%$ total $)$ \\
\hline$S l c 8 b 1^{\text {fl/fl }}$ & $91(50.3)$ & $90.5(50)$ \\
\hline Slc $8 b 1^{\mathrm{fl} / f l} \times$ aMHC-Cre & $90(49.7)$ & $90.5(50)$ \\
\hline Total for 17 litters: & 181 & 181 \\
\hline
\end{tabular}

Resultant genotypes for pups 3 weeks of age born from $S / c 8 b 1^{f / f}$; a MHC-Cre (heterozygous for Cre transgene) $\times$ $S I c 8 b 1^{f / f}$ breeder pairs. All knockouts were born at expected Mendelian ratios with no diference in survival.

\section{Supplementary Material}

Refer to Web version on PubMed Central for supplementary material.

\section{Acknowledgments}

We thank T. Tierney, N. Shah, and P. Kelly for technical assistance in the Elrod Laboratory. We thank S. Modla for her expertise in TEM sample processing (Delaware Biotechnology Institute). This study was supported by grants to J.W.E. from the NIH (R01 HL123966, P01 DA037830 sub-8614) and AHA (14SDG18910041) and (15PRE25080299 to T.S.L.), (16PRE31030038 to A.A.L.), and (17PRE33460423 to J.P.L.).

\section{References}

1. Murphy E, et al. Unresolved questions from the analysis of mice lacking MCU expression. Biochem Biophys Res Commun. 2014; 449:384-385. [PubMed: 24792186]

2. Palty R, et al. NCLX is an essential component of mitochondrial $\mathrm{Na}^{+} / \mathrm{Ca}^{2+}$ exchange. Proc Natl Acad Sci USA. 2010; 107:436-441. [PubMed: 20018762]

3. Sekler I. Standing of giants shoulders the story of the mitochondrial $\mathrm{Na}^{+} \mathrm{Ca}^{2+}$ exchanger. Biochem Biophys Res Commun. 2015; 460:50-52. [PubMed: 25998733]

4. Antony AN, et al. MICU1 regulation of mitochondrial $\mathrm{Ca}^{2+}$ uptake dictates survival and tissue regeneration. Nat Commun. 2016; 7:10955. [PubMed: 26956930]

5. Mallilankaraman K, et al. MICU1 is an essential gatekeeper for MCU-mediated mitochondrial $\mathrm{Ca}^{2+}$ uptake that regulates cell survival. Cell. 2012; 151:630-644. [PubMed: 23101630] 
6. Mallilankaraman $\mathrm{K}$, et al. MCUR1 is an essential component of mitochondrial $\mathrm{Ca}^{2+}$ uptake that regulates cellular metabolism. Nat Cell Biol. 2012; 14:1336-1343. [PubMed: 23178883]

7. De Stefani D, Raffaello A, Teardo E, Szabò I, Rizzuto R. A forty-kilodalton protein of the inner membrane is the mitochondrial calcium uniporter. Nature. 2011; 476:336-340. [PubMed: 21685888]

8. Baughman JM, et al. Integrative genomics identifies MCU as an essential component of the mitochondrial calcium uniporter. Nature. 2011; 476:341-345. [PubMed: 21685886]

9. Perocchi F, et al. MICU1 encodes a mitochondrial EF hand protein required for $\mathrm{Ca}^{2+}$ uptake. Nature. 2010; 467:291-296. [PubMed: 20693986]

10. Sancak Y, et al. EMRE is an essential component of the mitochondrial calcium uniporter complex. Science. 2013; 342:1379-1382. [PubMed: 24231807]

11. Bers DM. Calcium cycling and signaling in cardiac myocytes. Annu Rev Physiol. 2008; 70:23-49. [PubMed: 17988210]

12. Maack C, O'Rourke B. Excitation-contraction coupling and mitochondrial energetics. Basic Res Cardiol. 2007; 102:369-392. [PubMed: 17657400]

13. Luongo TS, et al. The mitochondrial calcium uniporter matches energetic supply with cardiac workload during stress and modulates permeability transition. Cell Reports. 2015; 12:23-34. [PubMed: 26119731]

14. Pan X, et al. The physiological role of mitochondrial calcium revealed by mice lacking the mitochondrial calcium uniporter. Nat Cell Biol. 2013; 15:1464-1472. [PubMed: 24212091]

15. Kwong JQ, et al. The mitochondrial calcium uniporter selectively matches metabolic output to acute contractile stress in the heart. Cell Reports. 2015; 12:15-22. [PubMed: 26119742]

16. Palty R, Sekler I. The mitochondrial $\mathrm{Na}^{+} / \mathrm{Ca}^{2+}$ exchanger. Cell Calcium. 2012; 52:9-15. [PubMed: 22430014]

17. Kostic M, et al. PKA phosphorylation of NCLX reverses mitochondrial calcium overload and depolarization, promoting survival of PINK1-deficient dopaminergic neurons. Cell Reports. 2015; 13:376-386. [PubMed: 26440884]

18. Skarnes WC, et al. A conditional knockout resource for the genome-wide study of mouse gene function. Nature. 2011; 474:337-342. [PubMed: 21677750]

19. Sohal DS, et al. Temporally regulated and tissue-specific gene manipulations in the adult and embryonic heart using a tamoxifen-inducible Cre protein. Circ Res. 2001; 89:20-25. [PubMed: 11440973]

20. Davis J, Maillet M, Miano JM, Molkentin JD. Lost in transgenesis: a user's guide for genetically manipulating the mouse in cardiac research. Circ Res. 2012; 111:761-777. [PubMed: 22935533]

21. Brookes PS, Yoon Y, Robotham JL, Anders MW, Sheu SS. Calcium, ATP, and ROS: a mitochondrial love-hate triangle. Am J Physiol Cell Physiol. 2004; 287:C817-C833. [PubMed: 15355853]

22. Baines CP, et al. Loss of cyclophilin D reveals a critical role for mitochondrial permeability transition in cell death. Nature. 2005; 434:658-662. [PubMed: 15800627]

23. Oka T, et al. Cardiac-specific deletion of Gata4 reveals its requirement for hypertrophy, compensation, and myocyte viability. Circ Res. 2006; 98:837-845. [PubMed: 16514068]

24. Boyman L, et al. Calcium movement in cardiac mitochondria. Biophys J. 2014; 107:1289-1301. [PubMed: 25229137]

25. Bers DM. Altered cardiac myocyte Ca regulation in heart failure. Physiology (Bethesda). 2006; 21:380-387. [PubMed: 17119150]

26. Kohlhaas $\mathrm{M}$, et al. Elevated cytosolic $\mathrm{Na}^{+}$increases mitochondrial formation of reactive oxygen species in failing cardiac myocytes. Circulation. 2010; 121:1606-1613. [PubMed: 20351235]

27. Liu T, O'Rourke B. Enhancing mitochondrial $\mathrm{Ca}^{2+}$ uptake in myocytes from failing hearts restores energy supply and demand matching. Circ Res. 2008; 103:279-288. [PubMed: 18599868]

28. Nakayama $\mathrm{H}$, et al. $\mathrm{Ca}^{2+}$ - and mitochondrial-dependent cardiomyocyte necrosis as a primary mediator of heart failure. J Clin Invest. 2007; 117:2431-2444. [PubMed: 17694179]

29. Holmström KM, et al. Assessment of cardiac function in mice lacking the mitochondrial calcium uniporter. J Mol Cell Cardiol. 2015; 85:178-182. [PubMed: 26057074] 
30. Williams GS, Boyman L, Lederer WJ. Mitochondrial calcium and the regulation of metabolism in the heart. J Mol Cell Cardiol. 2015; 78:35-45. [PubMed: 25450609]

31. Reynolds ES. The use of lead citrate at high $\mathrm{pH}$ as an electron-opaque stain in electron microscopy. J Cell Biol. 1963; 17:208-212. [PubMed: 13986422]

32. Zhang $\mathrm{X}$, et al. Persistent increases in $\mathrm{Ca}^{2+}$ influx through Cav1.2 shortens action potential and causes $\mathrm{Ca}^{2+}$ overload-induced afterdepolarizations and arrhythmias. Basic Res Cardiol. 2016; 111:4. [PubMed: 26611208]

33. Frezza C, Cipolat S, Scorrano L. Organelle isolation: functional mitochondria from mouse liver, muscle and cultured fibroblasts. Nat Protocols. 2007; 2:287-295. [PubMed: 17406588]

34. Hamer PW, McGeachie JM, Davies MJ, Grounds MD. Evans Blue Dye as an in vivo marker of myofibre damage: optimising parameters for detecting initial myofibre membrane permeability. $\mathrm{J}$ Anat. 2002; 200:69-79. [PubMed: 11837252]

35. Dipla K, Mattiello JA, Jeevanandam V, Houser SR, Margulies KB. Myocyte recovery after mechanical circulatory support in humans with end-stage heart failure. Circulation. 1998; 97:2316-2322. [PubMed: 9639375]

36. Gao E, et al. A novel and efficient model of coronary artery ligation and myocardial infarction in the mouse. Circ Res. 2010; 107:1445-1453. [PubMed: 20966393] 

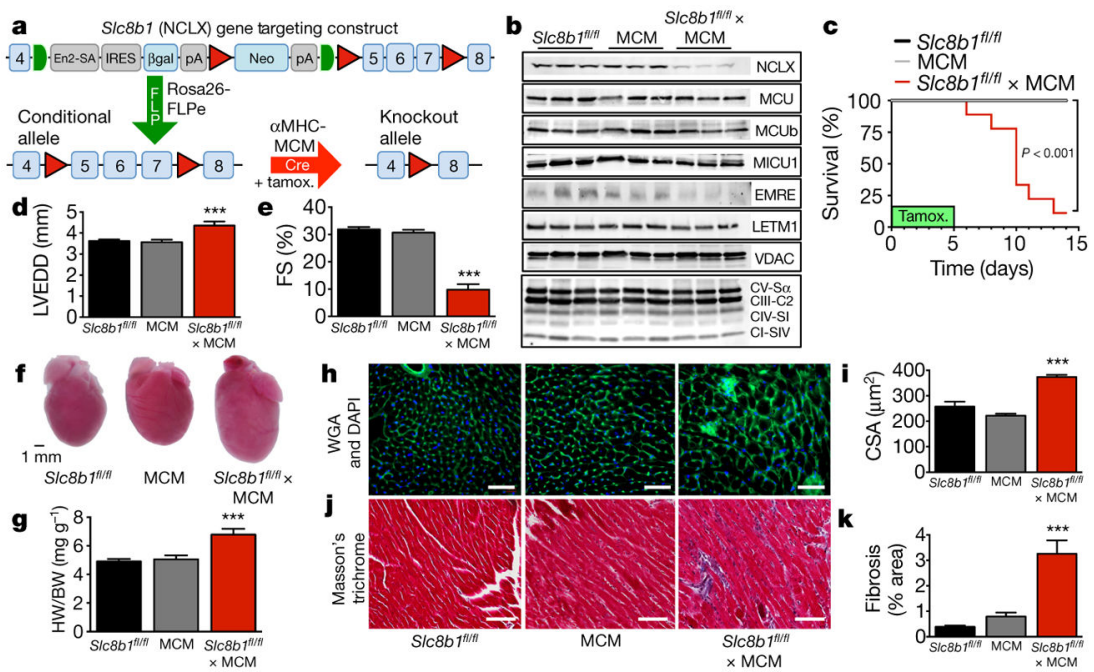

Figure 1. Deletion of $S l c 8 b 1$ from adult cardiomyocytes causes left ventricular remodelling and heart failure followed by sudden death

a, Schematic of gene targeting strategy. $\operatorname{Lox} \mathrm{s}$ sites (red triangles) and FRT sites (green semicircles) flank a splice acceptor site (En2-SA), $\beta$-galactosidase ( $\beta$ gal) reporter, and neomycin resistance (Neo) cassette. Mutant founders were crossed with Rosa26-FLPe mice for removal of FRT-flanked region. $S / c 8 b 1^{f l / f l}$ were crossed with cardiomyocyte-restricted (aMHC promoter), tamoxifen-inducible MCM transgenic mice. All mice were treated with tamoxifen (tamox.) for 5 days. All phenotyping was conducted 3 days post tamoxifen treatment. b, Western blots for NCLX expression and other proteins associated with $\mathrm{mCa}^{2+}$ exchange. MCU, mitochondrial calcium uniporter; MCUb, mitochondrial calcium uniporter $\beta$ subunit; MICU1, mitochondrial calcium uptake 1; EMRE, essential MCU regulator; LETM1, leucine zipper and EF-hand-containing transmembrane protein 1. Voltage dependent anion channel (VDAC) and oxidative phosphorylation components were used as mitochondrial loading controls. CV-Sa, complex V a subunit; CIII-C2, complex III-core protein 2; CIV-SI, complex IV subunit I; CI-SIV, complex I subunit NDUFB8. $n=3$ mice per group. c, Kaplan-Meier survival curves after tamoxifen treatment. $n=14 S / c 8 b 1^{f l / f 1}, n=9$ MCM, $n=9 S l c 8 b 1^{f l / f l} \times$ MCM. d, e, Echocardiography of left ventricular structure and function 3 days post tamoxifen treatment. $n=13$ Slc8b1 $1^{f l / f l}, n=9 \mathrm{MCM}, n=6$ Slc8b1 $1^{f l / f l}$ $\times$ MCM. d, Left ventricular end-diastolic dimension (LVEDD). e, Per cent fractional shortening (FS). f, Gross heart images 3 days post tamoxifen. g, Heart weight to body weight ratio (HW/BW, $n=5-7$ mice per group). $\mathbf{h}$, Images of left ventricular tissue stained with wheat germ agglutinin (WGA, green) to delineate sarcolemma and DAPI (blue). Scale bars, $50 \mu \mathrm{m}$. i, Cardiomyocyte cross-sectional area (CSA). Scale bar, $400 \mu \mathrm{m} ; n=3-4$ mice per group). $\mathbf{j}$, Masson's trichrome staining of left ventricular tissue to evaluate collagen deposition (blue). Scale bars, $400 \mu \mathrm{m}$. $\mathbf{k}$, Quantification of fibrotic area as a percentage of left ventricle. $n=16$ Slc8b1 $1^{f l / f 1}, n=13 \mathrm{MCM}, n=10$ Slc8b1 $1^{f l / f 1} \times \mathrm{MCM}$; *** $P<0.001$. 

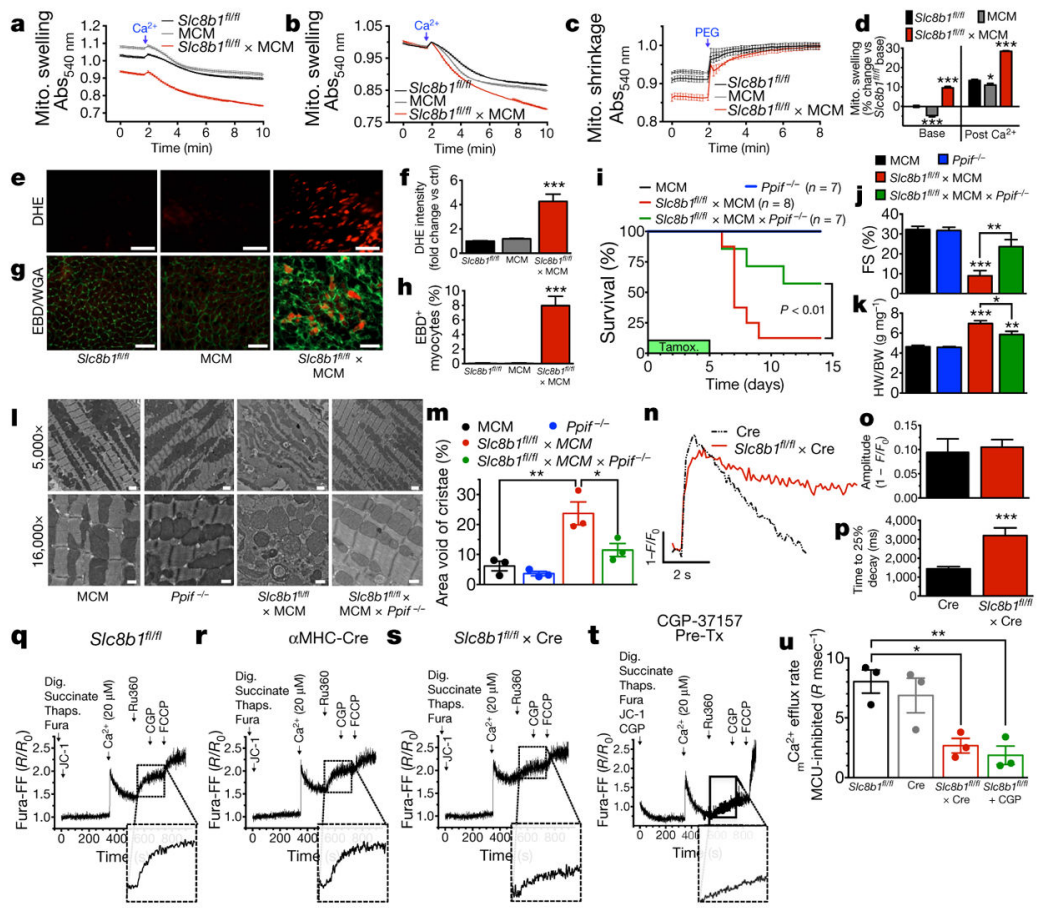

$\alpha M H C-C r e \mathbf{s} S / C 8 b^{1 / H} \times$ Cre $\mathbf{t}$

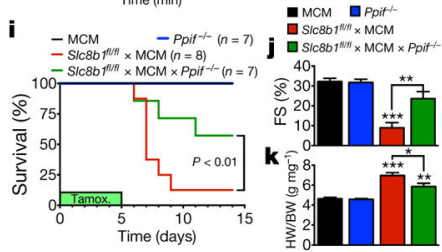

n
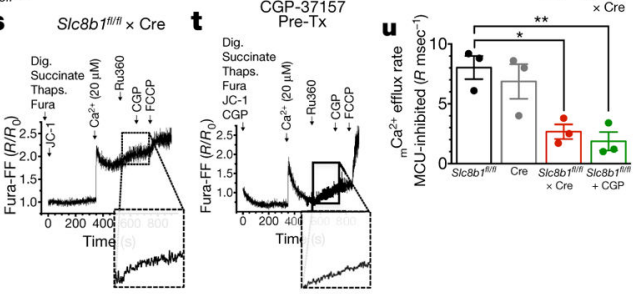

Figure 2. Deletion of $S l c 8 b 1$ in adult mice ablates $\mathrm{m}^{\mathrm{Ca}^{2+}}$ efflux resulting in mitochondrialdependent necrotic cell death

a, Mitochondrial swelling induced by $500 \mu \mathrm{M} \mathrm{Ca}^{2+}$ ( $n=4$ per group). b, Mitochondrial swelling normalized before $\mathrm{Ca}^{2+}$ addition ( $n=4$ per group). $\mathbf{c}$, Mitochondrial shrinkage induced by PEG ( $n=4$ per group). d, Per cent change in swelling versus control. e, Images of dihydroethidium (DHE) staining in live left ventricular tissue. Scale bars, $40 \mu \mathrm{m} ; n=10$ $S l c 8 b 1^{f l / f l}, n=8 \mathrm{MCM}, n=19$ Slc $8 b 1^{f l / f l} \times \mathrm{MCM}$. f, Fold-change in DHE signal versus control. g, Evans blue dye (EBD) and wheat germ agglutinin (WGA) staining in left ventricular tissue 3 days post tamoxifen. Scale bars, $50 \mu \mathrm{m}$. h, Per cent EBD ${ }^{+}$ cardiomyocytes. $n=15$ mice per group. i, Kaplan-Meier survival curves of mice during tamoxifen treatment. $n=7$ Ppif $^{-/}, 10 \mathrm{MCM}, 8$ Slc $8 b 1^{f l / f l} \times \mathrm{MCM}, 8$ Slc $8 b 1^{f l / f l} \times \mathrm{MCM}$ $\times$ Ppif $^{\prime-}$. $\mathbf{j}$, Per cent fractional shortening (FS) 3 days post tamoxifen administration. $n=5-6$ mice per group. $\mathbf{k}$, Heart weight to body weight ratio 3 days post tamoxifen treatment. $n=5$ mice per group. l, Electron microscopy images of left ventricular tissue 3 days after tamoxifen treatment, imaged at 5,000x magnification (scale bars, $1 \mu \mathrm{m}$ ) and 16,000x magnification (scale bars, $500 \mathrm{~nm}$ ). m, Mitochondrial cristae density, represented as per cent area void of cristae. $n=3-4$ mice per group. $\mathbf{n}$, Mitycam $\mathrm{mCa}^{2+}$ transients of ACMs paced at $0.1 \mathrm{~Hz} . F / F_{0}$ indicates the maximum fluorescence of the cell over the average fluorescence before stimulation. $\mathbf{o},{ }_{\mathrm{m}} \mathrm{Ca}^{2+}$ peak amplitude. $\mathbf{p}, \mathrm{mCa}^{2+}$ time to $25 \%$ decay. $n=7 \mathrm{Cre}, n=14$ Slc $8 b 1^{f l / f l} \times$ Cre ACMs. $\mathbf{q}-\mathbf{s},{ }_{m} \mathrm{Ca}^{2+}$ uptake and efflux in isolated, permeabilized ACMs. dig., digitonin; thaps., thapsigargin; Ru360, MCU inhibitor; CGP, NCLX inhibitor. Inset, magnified smoothed efflux tracing. $R$ indicates the ratio of the ratiometric reporter Fura-FF ( $340 / 380 \mathrm{~nm}$ excitation and $510 \mathrm{~nm}$ emission). $R / R_{0}$ indicates the ratio at each time point over the ratio at time $0 . \mathbf{t}$, Wild-type ACMs pre-treated with NCLX inhibitor. $\mathbf{u}, \mathrm{m}^{2+}$ efflux rate. $n=3$ replicates per group; $* P<0.05, * * P<0.01, * * * P<0.001$. 

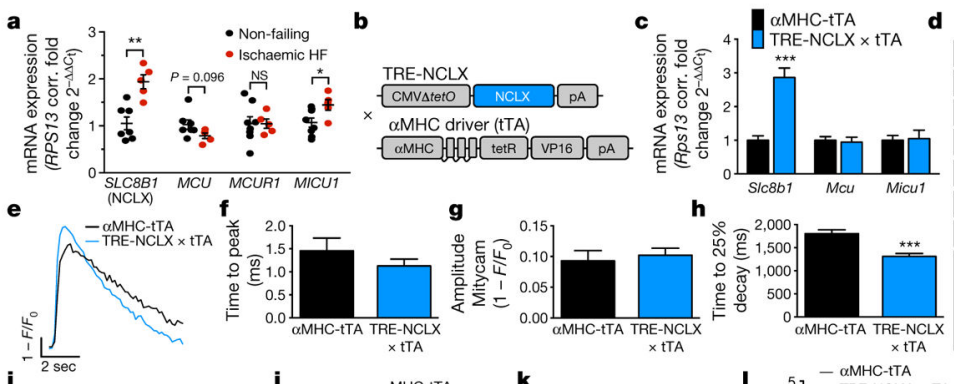

d $\underline{\triangle M H C-T T A}$ TRE-mNCX $\times$ TTA

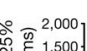
을

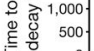

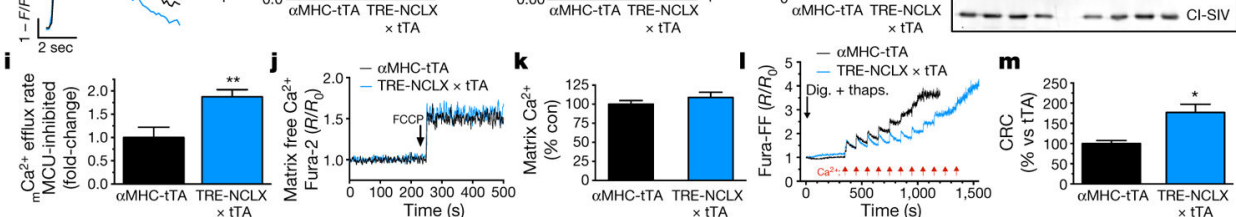

Figure 3. Cardiomyocyte overexpression of NCLX increases $\mathrm{mCa}^{2+}$ efflux capacity a, mRNA expression of $\mathrm{m}^{2+}{ }^{2+}$ exchange genes in left ventricular tissue of patients with ischaemic heart failure (HF). Fold change versus non-failing controls. $n=7$ non-failing and $n=5$ ischaemic heart failure). b, Schematic of transgenic construct for conditional, cardiacrestricted NCLX-overexpressing mouse. $\mathbf{c}$, Cardiac mRNA expression of $\mathrm{m}^{2 a^{2+}}$ exchange genes, fold-change versus tTA control. $n=4$ mice per group. d, Western blots of cardiac NCLX expression and other ${ }_{\mathrm{m}} \mathrm{Ca}^{2+}$ exchange proteins. $n=4-8$ mice per group. $\mathbf{e}$, Mitycam $\mathrm{mCa}^{2+}$ transients in ACM paced at $0.1 \mathrm{~Hz}$. f, $\mathrm{mCa}^{2+}$ time to peak intensity. $\mathbf{g}, \mathrm{m}^{2+}$ peak amplitude. $\mathbf{h},{ }_{\mathrm{m}} \mathrm{Ca}^{2+}$ time to $25 \%$ decay. $n=6-11$ ACMs per group. $\mathbf{i}, \mathrm{m}_{\mathrm{m}}{ }^{2+}$ efflux independent of uptake, fold change versus tTA control. $\mathbf{j}$, Recording of ${ }_{\mathrm{m}} \mathrm{Ca}^{2+}$ content before and after FCCP to release matrix free $\mathrm{Ca}^{2+}$. $\mathbf{k}$, Per cent change in matrix $\mathrm{Ca}^{2+}$ content. $\mathbf{l}, \mathrm{mCa}^{2+}$ retention capacity (CRC) in isolated ACMs. $\mathbf{m}$, Quantification of CRC as per cent change versus tTA control. $n=3$ replicates per group; $* P<0.05$, ** $P<0.01$, *** $P<$ 0.001 ; NS, not significant. 

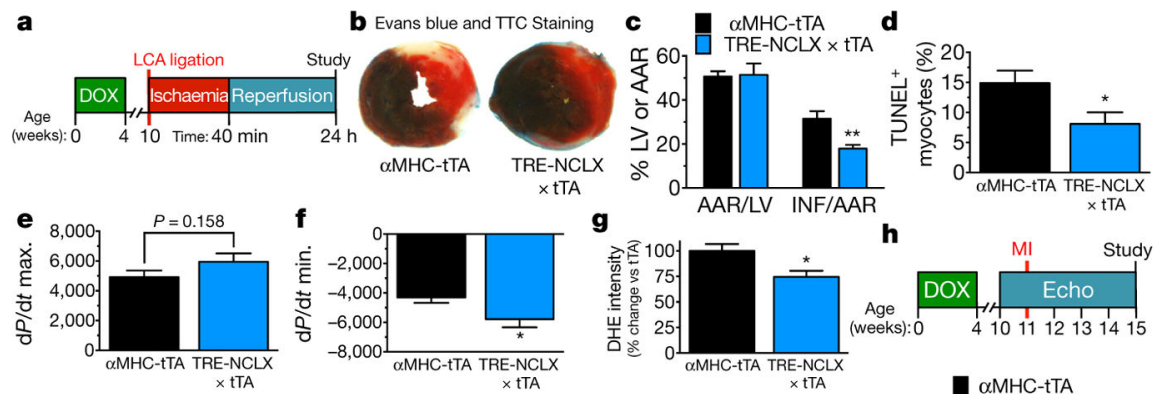

$\mathbf{g}$
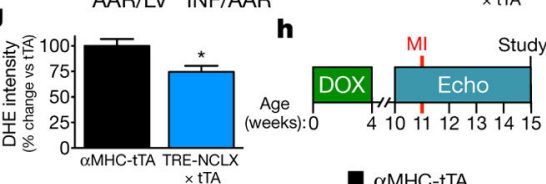

i

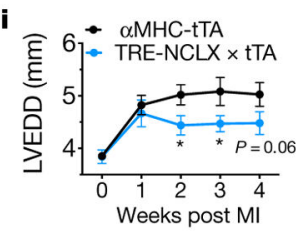

j $-\alpha M H C-t T A$

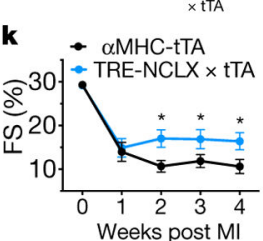

$\alpha M H C-$-tTA
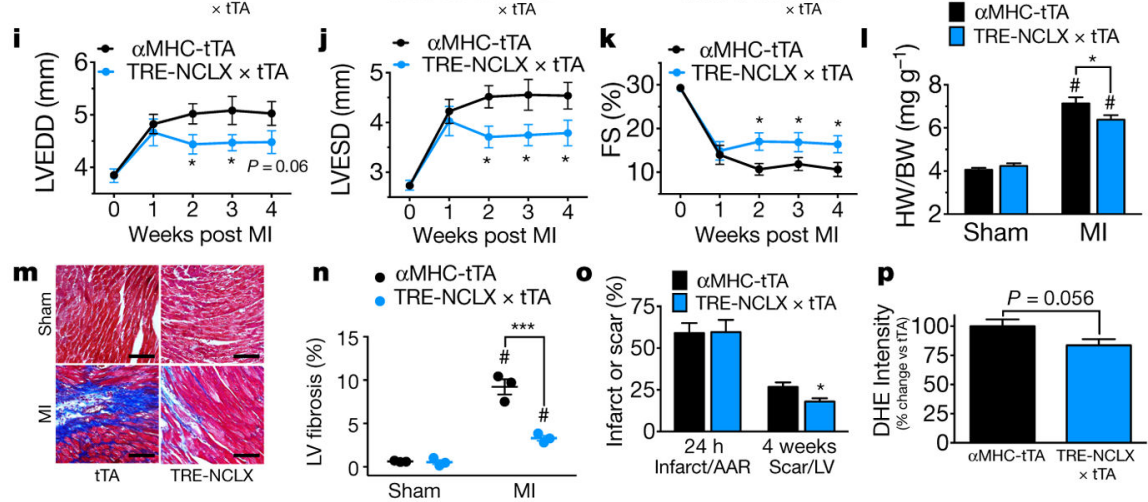

Figure 4. Overexpression of NCLX protects against myocardial ischaemic reperfusion injury and ischaemic heart failure

a, Timeline of myocardial ischaemia reperfusion experimental protocol. DOX, doxycycline administration. b, Mid-ventricular cross-sections of hearts $24 \mathrm{~h}$ after reperfusion and EBD perfusion and TTC staining (non-blue area indicates the area at risk (AAR); red area, viable myocardium; white area, infarct). c, Per cent AAR of the left ventricle and per cent infarct of the AAR. $n=11$ mice per group. $\mathbf{d}$, TUNEL ${ }^{+}$cardiomyocytes post ischaemia reperfusion. $n$ $=5$ mice per group. $\mathbf{e}, \mathbf{f}$, Invasive haemodynamics post ischaemia reperfusion. $\mathbf{e}, \mathrm{d} P / \mathrm{d} t$ maximum (contractility). f, $\mathrm{d} P / \mathrm{d} t$ minimum (relaxation). $n=13-16$ mice per group. $\mathbf{g}$, DHE staining in live myocardium $24 \mathrm{~h}$ post ischaemia reperfusion. $n=6 \mathrm{tTA}$ and $n=5$ NCLX-Tg. h, Timeline of myocardial infarction (MI) experimental protocol. Echo, left ventricular echocardiography. i, Left-ventricular end-diastolic dimension (LVEDD). j, Left-ventricular end-systolic dimension (LVESD). k, Per cent fractional shortening (FS). $n=19$ tTA, $n=16$ NCLX-Tg. 1, Heart weight to body weight ratio. $n=6$, sham tTA, $n=7$ sham NCLX-Tg, $n$ $=17$ tTA, $n=17$ NCLX-Tg. m, Images of Masson's trichrome-stained left ventricular sections at the border zone 4 weeks post myocardial infarction. Scale bars, $400 \mu \mathrm{m}$. $\mathbf{n}$, Per cent fibrosis in peri-infarct and remote regions of the left ventricle 4 weeks post infarction. $n$ $=3$ mice per group. $\mathbf{o}$, Per cent infarct of AAR $24 \mathrm{~h}$ after LCA ligation and scar of left ventricle 4 weeks post infarction. $n=5$ mice per group. p. DHE staining in live myocardium 4 weeks post infarction. $n=8-9$ mice per group. $* P<0.05$, $* * P<0.01$, *** $P<0.001$; \#P< 0.05 versus sham control $(\mathbf{l}, \mathbf{n})$. 